\title{
DYNAMICAL TRANSITIONS OF TURING PATTERNS
}

\author{
HANS G. KAPER, SHOUHONG WANG, AND MASOUD YARI
}

\begin{abstract}
This article is concerned with the formation and persistence of spatiotemporal patterns in binary mixtures of chemically reacting species, where one of the species is an activator, the other an inhibitor of the chemical reaction. The system of reaction-diffusion equations is reduced to a finite system of ordinary differential equations by a variant of the center-manifold reduction method. The reduced system fully describes the local dynamics of the original system near transition points at the onset of instability. The attractor-bifurcation theory is used to give a complete characterization of the bifurcated objects in terms of the physical parameters of the problem. The results are illustrated for the Schnakenberg model.
\end{abstract}

\section{INTRODUCTION}

This article is concerned with the formation and persistence of spatiotemporal patterns in binary mixtures of chemically reacting species, where one of the species is an activator, the other an inhibitor of the chemical reaction. Typically, these patterns arise when the system transits from one stable equilibrium to another.

The phenomenon of pattern formation in activator-inhibitor systems is commonly associated with the name of Turing, who showed in his pioneering study of morphogenesis [20] that structure can emerge from a structureless state without the apparent action of an external organizing force as a result of a competition between reaction and diffusion. Experimental evidence for the existence of so-called Turing patterns in chemistry is described in, among others, Refs. $[4,5]$. The impact of Turing's work on the theory of pattern formation in biology is discussed, for example, in Ref. [12]. Turing's ideas have penetrated well beyond the fields of biology and chemistry; for example, an application to fingerprint imaging is given in Ref. [16]. A general overview of the theory of pattern formation can be found in the review article by Cross and Hohenberg [3] and the recent text by Hoyle [8].

Linear stability analysis has been a basic tool in the study of reactiondiffusion systems. It provides insight into the nonlinear behavior of such systems as well, since the latter can often be approximated, at 
least for brief lengths of time, by linearized systems. But as time evolves, the nonlinear structure takes over, and other tools are needed to study the long-time behavior. A weakly nonlinear stability analysis can be used to identify the steady states and their connecting orbits after a bifurcation. Such an analysis can be justified rigorously on the basis of modulation theory and a Ginzburg-Landau approximation $[2,6,18,19,21]$. A survey of weakly nonlinear analyses for reaction-diffusion systems can be found, for example, in Ref. [22]. Murray's monograph [13] discusses applications to biological systems such as animal coat patterns. Sometimes, special techniques have been applied to the study of Turing patterns in different regimes. For example, Ref. [9] deals with the stability of symmetric $N$-peaked steady states for systems where the inhibitor diffuses much more rapidly than the activator. We also mention Refs. [1,14,15], which deal with the Schnakenberg model on heterogeneous domains, where spatially varying diffusion coefficients may prevent the degeneracy of a Turing bifurcation.

In this article we present a different nonlinear approach to the instabilities and transitions in Turing systems. By using a finite-dimensional approximation to the system of reaction-diffusion equations in certain unstable domains we are able to give a complete characterization of the attractors and their basins of attraction in terms of the physical parameters of the problem. The two essential elements are a new approach to the center-manifold reduction and an application of the attractorbifurcation theory of Ref. [10]. The center-manifold reduction projects the original system of partial differential equations to a set of ordinary differential equations that describe the leading-order dynamics in the neighborhood of a transition point. The reduced system retains the essential features of the dynamics, is much simpler than the original system, and yields explicitly computable quantities that completely characterize the transitions of the system.

Certain computations simplify when symmetries are present, but we emphasize that the method presented here is essentially independent of such symmetries and applies equally well to domains without symmetries. Common bifurcation theory, which relies on the presence of symmetries, represents attractors in terms of steady states and their connecting orbits but overlooks many transient states that can be important from the viewpoint of applications. The existence of transient states in activator-inhibitor systems and their importance in biological systems are discussed, for example, in Refs. [23, 24]. The approach presented in this paper yields complete information about bifurcations, transitions, stability, and persistence, including information about transient states, in terms of the physical parameters of the 
system. To the best of our knowledge, no other approach matches the comprehensive nature of the results obtained by attractor-bifurcation theory. Since there are, in fact, instances of pattern formation (for example, quasi-patterns) that cannot be described in the framework of equivariant bifurcation theory, there is an obvious need to develop new methods to study bifurcations and transitions. The fact that the attractor-bifurcation method goes well beyond the usual symmetrybifurcation method has been demonstrated in other situations as well [10].

In this article we consider reaction-diffusion systems of the activatorinhibitor type on bounded domains. If the system is one-dimensional or two-dimensional and rectangular (nonsquare), we show that the bifurcated object consists of two points, each with its basin of attraction (Theorem 4.2, Fig. 2). In the case of a square domain, the primary bifurcation is either a pitchfork bifurcation or an $S^{1}$ bifurcation, depending on the parameters of the problem. In the case of an $S^{1}$-bifurcation, the phase diagram after bifurcation contains either an infinite number of steady-state solutions or eight steady-state solutions and the heteroclinic orbits connecting them (Theorem 5.2, Fig. 3). Specific criteria that determine the nature of the transition when the first unstable mode arises are given in terms of eigenvalues and eigenvectors and are thus related directly to the physical parameters of the problem.

Although the focus in this article is on activator-inhibitor systems, the analysis is general and applies, for example, also to systems consisting of a self-amplifying activator and a depleted substrate.

Following is an outline of the paper. In Section 2, we formulate the reaction-diffusion problem for an activator-inhibitor mixture and rewrite it as an evolution equation in a function space. In Section 3, we study the exchange of stability, which is crucial for the stability and bifurcation analysis. The results of the bifurcation analysis for the one-dimensional case are given in Section 4 and for the two-dimensional case in Section 5. In Section 6, we illustrate the theoretical results on the Schnakenberg equation. Section 7 summarizes the conclusions. In Appendix A we give a brief summary of the reduction method and the attractor-bifurcation theory.

\section{Statement of the Problem}

Turing's theory of pattern formation refers to a mixture of two chemical species that simultaneously react and diffuse; one of the species is an activator, the other an inhibitor of the chemical reaction. The concentrations $U$ and $V$ of the activator and inhibitor satisfy a system of 
reaction-diffusion equations,

$$
\begin{aligned}
& U_{t}=d_{1} \Delta U+f(U, V), \\
& V_{t}=d_{2} \Delta V+g(U, V),
\end{aligned}
$$

subject to given boundary and initial conditions. Here, $\Delta$ denotes the Laplacian associated with diffusion of the species, $d_{1}$ and $d_{2}$ are diffusion coefficients that are constant and positive, and $f$ and $g$ are nonlinear functions describing the kinetics of the chemical reaction. The functions $f$ and $g$ are such that

$$
f(\bar{u}, \bar{v})=0, \quad g(\bar{u}, \bar{v})=0
$$

for some positive constants $\bar{u}$ and $\bar{v}$, so $(\bar{u}, \bar{v})$ is a uniform steady-state solution of Eq. (2.1). We assume that this equilibrium solution is stable in the absence of diffusion. Wre interested in solutions that bifurcate from it and, in particular, in the long-term dynamics of the bifurcating solutions.

Before proceeding to the bifurcation analysis we rescale time and space and rewrite the system (2.1) in the form

$$
\begin{aligned}
& U_{t}=\Delta U+\gamma f(U, V), \\
& V_{t}=d \Delta V+\gamma g(U, V),
\end{aligned}
$$

where $\gamma=1 / d_{1}$ and $d=d_{2} / d_{1}$. Thus, $\gamma$ is a measure of the ratio of the characteristic times for diffusion and chemical reaction, and $d$ is the ratio of the diffusion coefficients of the two species. We assume that the system (2.3) is satisfied on an open bounded domain $\Omega \subset \mathbf{R}^{n}(n=1,2)$ and that $U$ and $V$ satisfy Neumann (no-flux) boundary conditions on the boundary $\partial \Omega$ of $\Omega$.

\subsection{Bifurcation Problem. Let}

$$
U=\bar{u}+u, \quad V=\bar{v}+v,
$$

where $\bar{u}$ and $\bar{v}$ satisfy the identities (2.2). Expanding $f$ and $g$ in their Taylor series around $(\bar{u}, \bar{v})$, we see that $u$ and $v$ satisfy a system of equations of the form

$$
\begin{aligned}
& u_{t}=\Delta u+\gamma\left(f_{u}(\bar{u}, \bar{v}) u+f_{v}(\bar{u}, \bar{v}) v\right)+\gamma f_{1}(u, v), \\
& v_{t}=d \Delta v+\gamma\left(g_{u}(\bar{u}, \bar{v}) u+g_{v}(\bar{u}, \bar{v}) v\right)+\gamma g_{1}(u, v) .
\end{aligned}
$$

The nonlinear functions $f_{1}$ and $g_{1}$ incorporate the higher-order terms in the Taylor expansions. Henceforth we omit the $\operatorname{arguments}(\bar{u}, \bar{v})$ and write $f_{u}$ for $f_{u}(\bar{u}, \bar{v})$, and so forth.

Since $U$ and $V$ are associated respectively with the activator and the inhibitor of the chemical reaction, $f_{u}$ and $g_{v}$ satisfy the inequalities

$$
f_{u}>0, g_{v}<0 \text {. }
$$


Furthermore, since the equilibrium solution $(\bar{u}, \bar{v})$ is stable in the absence of diffusion,

$$
f_{u} g_{v}-f_{v} g_{u}>0, \quad f_{u}+g_{v}<0 .
$$

The first inequality, together with (2.6), implies that $f_{v} g_{u}<0$.

Henceforth we represent the two parameters $\gamma$ and $d$ by a single symbol, $\lambda=(\gamma, d)$, and consider $\lambda$ as the bifurcation parameter. We are interested in solutions of the system of Eqs. (2.5) that bifurcate from the trivial solution $(u, v)=(0,0)$ as $\lambda$ varies in the positive quadrant of the $(\gamma, d)$-plane.

2.2. Abstract Evolution Equation. The system of Eqs. (2.5) defines an abstract evolution equation for a vector-valued function $w$ : $t \mapsto w(t) \in H=\left(L^{2}(\Omega)\right)^{2}$,

$$
w(t)=\left(\begin{array}{c}
u(\cdot, t) \\
v(\cdot, t)
\end{array}\right), \quad t \geq 0 .
$$

Let $A: \operatorname{dom}(A) \rightarrow H$ be defined by the expression

$$
A=-\Delta I=\left(\begin{array}{cc}
-\Delta & 0 \\
0 & -\Delta
\end{array}\right)
$$

on $\operatorname{dom}(A)=H_{1}=\left\{w \in\left(H^{2}(\Omega)\right)^{2}: n \cdot \nabla w=0\right.$ on $\left.\partial \Omega\right\}$. Here, $H^{2}(\Omega)$ is the usual Sobolev space, and the gradient $\nabla w$ is taken componentwise. Let $B$ and $D$ be the linear operators in $H$ represented by the constant matrices

$$
B=\left(\begin{array}{ll}
f_{u} & f_{v} \\
g_{u} & g_{v}
\end{array}\right), \quad D=\left(\begin{array}{ll}
1 & 0 \\
0 & d
\end{array}\right) .
$$

Then the linear terms in Eq. (2.5) correspond to the operator

$$
L_{\lambda}=-A D+\gamma B \text {. }
$$

Next, let $G_{\lambda}: H \rightarrow H$ represent the nonlinear terms in Eq. (2.5),

$$
G_{\lambda}: w=\left(\begin{array}{c}
u \\
v
\end{array}\right) \mapsto G_{\lambda}(w)=\gamma\left(\begin{array}{c}
f_{1}(u, v) \\
g_{1}(u, v)
\end{array}\right) .
$$

Then Eq. (2.5) corresponds to the abstract evolution equation

$$
\frac{d w}{d t}=L_{\lambda} w+G_{\lambda}(w)
$$

for $w(t)$ in $H, t>0$. Equations of this type have been analyzed in detail by Ma and Wang; the relevant results from Ref. [10] are summarized in the Appendix (Section A.2). 
We assume that $G_{\lambda}$ can be written as the sum of symmetric multilinear forms,

$$
G_{\lambda}(w)=\sum_{k=2}^{\infty} G_{\lambda, k}(w, \ldots, w),
$$

where $G_{k}$ is a symmetric $k$-linear form $(k=2,3, \ldots)$. When the $k$ arguments of $G_{k}$ coincide, we write $G_{k}$ with a single argument, $G_{k}(w)=$ $G_{k}(w, \ldots, w)$.

\section{Exchange of Stability}

The inequalities (2.7) imply that

$$
\operatorname{det}(B)>0, \quad \operatorname{tr}(B)<0 .
$$

Under these conditions, diffusion has a destabilizing effect. At some critical value $\lambda_{0}$ of $\lambda$, the trivial solution of Eq. (2.5) loses stability and a bifurcation occurs.

3.1. Eigenvalues and Eigenvectors of $L_{\lambda}$ and $L_{\lambda}^{*}$. The negative Laplacian $-\Delta$ on a bounded domain $\Omega \in \mathbf{R}^{n}$ with Neumann boundary conditions is self-adjoint and positive in $L^{2}(\Omega)$. Its spectrum is discrete, consisting of eigenvalues $\rho_{k}$ with corresponding eigenvectors $\varphi_{k}$,

$$
-\Delta \varphi_{k}=\rho_{k} \varphi_{k}, \quad k=1,2, \ldots
$$

We assume that the eigenvalues are ordered, $0<\rho_{1} \leq \rho_{2} \leq \cdots$ and that the eigenvectors $\left\{\varphi_{k}\right\}_{k}$ form a basis in $L^{2}(\Omega)$.

It follows from the definition (2.9) that $A$ is self-adjoint and positive in $H$; its spectrum is also discrete, consisting of the same eigenvalues $\rho_{k}$ and the eigenvectors $\varphi_{k}$ once repeated. The operator $L_{\lambda}$ is reduced by projection to its components on the linear span of each eigenvector of $A$. Let $E_{k}$ be the component of $L_{\lambda}$ in the eigenspace associated with the eigenvalue $\rho_{k}$,

$$
E_{k}(\lambda)=-\rho_{k} D+\gamma B, \quad k=1,2, \ldots .
$$

The determinant and trace of $E_{k}(\lambda)$ are

$$
\begin{aligned}
\operatorname{det}\left(E_{k}(\lambda)\right) & =\gamma^{2} \operatorname{det}(B)+\gamma \rho_{k}\left|g_{v}\right|-\rho_{k} d\left(\gamma f_{u}-\rho_{k}\right), \\
\operatorname{tr}\left(E_{k}(\lambda)\right) & =\gamma \operatorname{tr}(B)-\rho_{k}(1+d) .
\end{aligned}
$$

Note that $\operatorname{tr}\left(E_{k}(\lambda)\right)$ is negative everywhere in the first quadrant and becomes more negative as $k$ increases.

The eigenvalues of $E_{k}(\lambda)$ come in pairs $\left(\beta_{k 1}, \beta_{k 2}\right)$,

$$
\beta_{k i}(\lambda)=\frac{1}{2} \operatorname{tr}\left(E_{k}(\lambda)\right) \pm\left(\left(\frac{1}{2} \operatorname{tr}\left(E_{k}(\lambda)\right)\right)^{2}-\operatorname{det}\left(E_{k}(\lambda)\right)\right)^{1 / 2}, \quad i=1,2 .
$$


The eigenvalues either are complex conjugate with $\Re \beta_{k 1}=\Re \beta_{k 2}<0$ or are both real with $\beta_{k 1}+\beta_{k 2}<0$. We identify $\beta_{k 1}$ with the upper $(+)$ sign and $\beta_{k 2}$ with the lower (-) sign, so $\Re \beta_{k 2} \leq \Re \beta_{k 1}$.

The eigenvector corresponding to the eigenvalue $\beta_{k i}$ of $L_{\lambda}$ is

$$
w_{k i}=\left(\begin{array}{c}
-\gamma f_{v} \varphi_{k} \\
\left(\gamma f_{u}-\rho_{k}-\beta_{k i}\right) \varphi_{k}
\end{array}\right) .
$$

The eigenvectors $w_{k 1}$ and $w_{k 2}$ are linearly independent as long as $\beta_{k 1} \neq$ $\beta_{k 2}$. The set of eigenvectors $\left\{w_{k i}\right\}_{k, i}$ forms a basis for $H$.

Note that $B$ is not symmetric; its adjoint $B^{*}$ is the transpose $B^{\prime}$ of $B$. Hence, the adjoint of $L_{\lambda}$ is $L_{\lambda}^{*}=-A D+\gamma B^{\prime}$, and the adjoint of $E_{k}(\lambda)$ is $E_{k}^{*}(\lambda)=-\rho_{k} D+\gamma B^{\prime}$. The eigenvalues $\beta_{k i}^{*}$ of $E_{k}^{*}(\lambda)$ are the complex conjugates of the eigenvalues $\beta_{k i}$ of $E_{k}(\lambda)$. Since the latter are either complex conjugate or real, the eigenvalues of $L_{\lambda}$ and $L_{\lambda}^{*}$ coincide; $\beta_{k 1}^{*}=\beta_{k 2}$, and $\beta_{k 2}^{*}=\beta_{k 1}$. The eigenvector corresponding to the eigenvalue $\beta_{k i}^{*}$ of $L_{\lambda}^{*}(\lambda)$ is

$$
w_{k i}^{*}=\left(\begin{array}{c}
-\gamma g_{u} \varphi_{k} \\
\left(\gamma f_{u}-\rho_{k}-\beta_{k i}^{*}\right) \varphi_{k}
\end{array}\right) \text {. }
$$

3.2. Exchange of Stability. The equation $\operatorname{det}\left(E_{k}(\lambda)\right)=0$ defines a curve $\Lambda_{k}$ in the $(\gamma, d)$-plane,

$$
\Lambda_{k}=\left\{(\gamma, d): \operatorname{det}\left(E_{k}(\lambda)\right)=0\right\}=\left\{(\gamma, d): d=d_{k}(\gamma)\right\}, \quad k=1,2, \ldots,
$$

where

$$
d_{k}(\gamma)=\frac{\gamma^{2} \operatorname{det}(B)-\gamma \rho_{k} g_{v}}{\rho_{k}\left(\gamma f_{u}-\rho_{k}\right)} .
$$

The expression for $d_{k}$ can be recast in the form

$$
d_{k}(\gamma)=\frac{\operatorname{det}(B)}{\rho_{k} f_{u}} \gamma-\frac{f_{v} g_{u}}{f_{u}^{2}}+\frac{\rho_{k} f_{v} g_{u}}{f_{u}^{2}\left(\gamma f_{u}-\rho_{k}\right)},
$$

which shows that $\Lambda_{k}$ has an oblique asymptote with slope $\operatorname{det}(B) /\left(\rho_{k} f_{u}\right)$ and a vertical asymptote at $\gamma_{k}=\rho_{k} / f_{u}$. Because of the inequalities (2.6) and (2.7), the slope of the oblique asymptote is positive and decreasing to zero as $k$ increases, and the vertical asymptote is in the right-half of the $(\gamma, d)$-plane and shifting to the right as $k$ increases. Moreover, if we rewrite the equation once more in the form

$$
d_{k}(\gamma)-d_{0}=\frac{\operatorname{det}(B)}{\rho_{k} f_{u}}\left(\gamma-\gamma_{k}\right)+\frac{\rho_{k}\left|f_{v} g_{u}\right|}{f_{u}^{3}}\left(\gamma-\gamma_{k}\right)^{-1}
$$

we see that $\Lambda_{k}$ is symmetric with respect to the point $\left(\gamma_{k}, d_{0}\right)$ where $\gamma_{k}=\rho_{k} / f_{u}$ and $d_{0}=\left(\operatorname{det}(B)+\left|f_{v} g_{u}\right|\right) / f_{u}^{2}$. The symmetry point is 
located in the first quadrant of the $(\gamma, d)$-plane; $\gamma_{k}$ increases as $k$ increases, and $d_{0}$ is independent of $k$. Therefore, each curve $\Lambda_{k}$ has a branch in the positive quadrant of the $(\gamma, d)$-plane. The curve $\Lambda_{k}$ separates the region where $\operatorname{det}\left(E_{k}(\lambda)\right)>0$ (below the curve) from the region where $\operatorname{det}\left(E_{k}(\lambda)\right)<0$ (above the curve). The positive branches of the first few curves are sketched in Fig. 1.

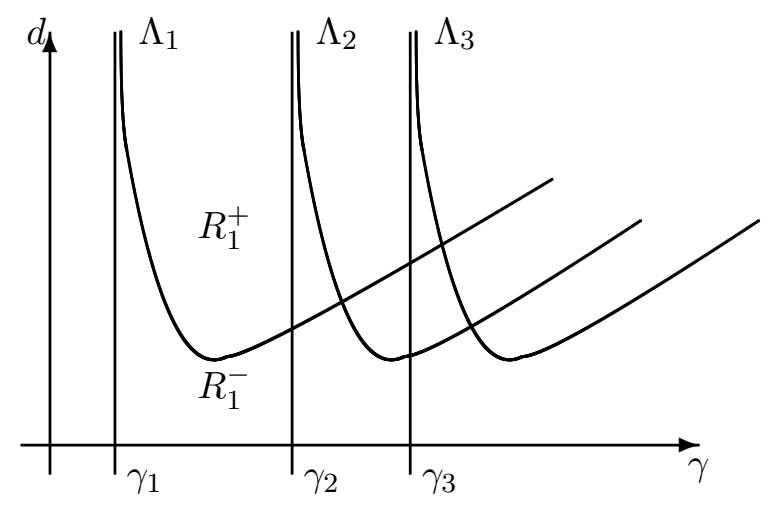

Figure 1. Positive branches of $\Lambda_{1}, \Lambda_{2}$, and $\Lambda_{3}$.

Consider the region in the positive quadrant bounded on the left by the vertical asymptote $\gamma_{k}=\rho_{k} / f_{u}$. The curve $\Lambda_{k}$ separates this region into two subregions,

$$
\begin{aligned}
& R_{k}^{-}=\left\{\lambda=(\gamma, d): \gamma>\gamma_{k}=\rho_{k} / f_{u}, 0<d<d_{k}(\gamma)\right\}, \\
& R_{k}^{+}=\left\{\lambda=(\gamma, d): \gamma>\gamma_{k}=\rho_{k} / f_{u}, d>d_{k}(\gamma)\right\} .
\end{aligned}
$$

The regions $R_{1}^{-}$and $R_{1}^{+}$are indicated in Fig. 1.

Lemma 3.1. The eigenvalues $\beta_{k i}(i=1,2)$ of $L_{\lambda}$ satisfy the inequalities

$$
\begin{array}{ll}
\Re \beta_{k 2}(\lambda) \leq \Re \beta_{k 1}(\lambda)<0 & \text { if } \lambda \in R_{k}^{-}, \\
\beta_{k 2}(\lambda)<0, \beta_{k 1}(\lambda)=0 & \text { if } \lambda \in \Lambda_{k}, \\
\beta_{k 2}(\lambda)<0, \beta_{k 1}(\lambda)>0 & \text { if } \lambda \in R_{k}^{+} .
\end{array}
$$

Furthermore, for all $j>k$,

$$
\Re \beta_{j 2}(\lambda) \leq \Re \beta_{j 1}(\lambda)<0 \text { if } \lambda \in \Lambda_{k} .
$$

Proof. In $R_{k}^{-}$, we have $\operatorname{tr}\left(E_{k}(\lambda)\right)<0$ and $\operatorname{det}\left(E_{k}(\lambda)>0\right.$. Hence, $\beta_{k 1}(\lambda)$ and $\beta_{k 2}(\lambda)$ either are complex conjugate with a negative real part or are both real and negative. On $\Lambda_{k}$, the leading eigenvalue $\beta_{k 1}(\lambda)$ is zero. Since $\operatorname{tr}\left(E_{k}(\lambda)\right)<0, \beta_{k 2}(\lambda)$ must be real and negative. In $R_{k}^{+}$, we have $\operatorname{tr}\left(E_{k}(\lambda)\right)<0$ and $\operatorname{det}\left(E_{k}(\lambda)<0\right.$, so $\beta_{k 1}(\lambda)$ and $\beta_{k 2}(\lambda)$ are both real, and they have opposite signs. 
On $\Lambda_{k}, \operatorname{det}\left(E_{k+1}(\lambda)\right)>0$. Since $\operatorname{det}\left(E_{k}(\lambda)\right)$ increases with $k$, it follows that $\operatorname{det}\left(E_{j}(\lambda)\right)>0$ for all $j>k$. Also, $\operatorname{tr}\left(E_{j}(\lambda)\right)<0$. Hence, $\beta_{j 1}(\lambda)$ and $\beta_{j 2}(\lambda)$ either are complex conjugate with a negative real part or are both real and negative.

The lemma implies that all eigenmodes are stable as long as $\lambda$ is below the curves $\Lambda_{k}$. As soon as an eigenvalue $\lambda$ crosses a curve $\Lambda_{k}$, however, the corresponding eigenmode becomes unstable, and an exchange of stability occurs. Without loss of generality, we will assume that the first exchange of stability occurs when $\lambda$ crosses the first curve, $\Lambda_{1}$, at some critical value $\lambda_{0}$, say.

\section{Bifurcation Analysis - One-Dimensional Domain}

Let $\Omega=(0, \ell)$. We reduce Eq. (2.13) to its center-manifold representation near $\lambda_{0}$. The main idea of the method is summarized in the Appendix (Section A.1).

The eigenvalues and eigenvectors of the negative Laplacian subject to Neumann boundary conditions (see Eq. (3.2)) are

$$
\rho_{k}=k^{2}(\pi / \ell)^{2}, \quad \varphi_{k}(x)=\cos \left(x \sqrt{ } \rho_{k}\right), x \in \Omega ; \quad k=1,2, \ldots
$$

The linear operator $L_{\lambda}$ decomposes into its components

$$
E_{k}(\lambda)=-\rho_{k} D+\gamma B, \quad k=1,2, \ldots,
$$

with

$$
\begin{aligned}
\operatorname{det}\left(E_{k}(\lambda)\right) & =\gamma^{2} \operatorname{det}(B)+\gamma \rho_{k}\left|g_{v}\right|-d \rho_{k}\left(\gamma f_{u}-\rho_{k}\right), \\
\operatorname{tr}\left(E_{k}(\lambda)\right) & =\gamma \operatorname{tr}(B)-(1+d) \rho_{k} .
\end{aligned}
$$

Each $E_{k}$ contributes two eigenvalues, $\beta_{k 1}$ and $\beta_{k 2}$, to the spectrum of $L_{\lambda}$; the expressions for $\beta_{k i}(i=1,2)$ in terms of $\operatorname{det}\left(E_{k}(\lambda)\right)$ and $\operatorname{tr}\left(E_{k}(\lambda)\right)$ are given in Eq. (3.5). The eigenvalues of the adjoint $L_{\lambda}^{*}$ are the complex conjugates $\left(\beta_{k 1}^{*}, \beta_{k 2}^{*}\right)=\left(\beta_{k 2}, \beta_{k 1}\right)$. The eigenvectors of $L_{\lambda}$ and $L_{\lambda}^{*}$ corresponding to the eigenvalues $\beta_{k i}$ and $\beta_{k i}^{*}$ are

$$
w_{k i}=\left(\begin{array}{c}
-\gamma f_{v} \cos \left(x \sqrt{ } \rho_{k}\right) \\
\left(\gamma f_{u}-\rho_{k}-\beta_{k i}\right) \cos \left(x \sqrt{ } \rho_{k}\right)
\end{array}\right)
$$

and

$$
w_{k i}^{*}=\left(\begin{array}{c}
-\gamma g_{u} \cos \left(x \sqrt{ } \rho_{k}\right) \\
\left(\gamma f_{u}-\rho_{k}-\beta_{k i}^{*}\right) \cos \left(x \sqrt{ } \rho_{k}\right)
\end{array}\right),
$$

respectively. 
4.1. Center-Manifold Reduction. In the region $R_{1}^{-}$, just below $\Lambda_{1}$ and sufficiently close to $\lambda_{0}$, both eigenvalues $\beta_{11}$ and $\beta_{12}$ are real, with $\beta_{12}<\beta_{11}<0$. As $\lambda$ approaches $\lambda_{0}$, the leading eigenvalue $\beta_{11}$ increases and, as $\lambda$ transits into $R_{1}^{+}, \beta_{11}$ passes through 0 and becomes positive.

Theorem 4.1. Near $\lambda_{0} \in \Lambda_{1}$, the solution of Eq. (2.13) can be expressed in the form

$$
w=y_{11} w_{11}+z, \quad z=y_{12} w_{12}+\sum_{k=2}^{\infty} \sum_{i=1,2} y_{k i} w_{k i},
$$

where the coefficient $y_{11}$ of the leading term satisfies the reduced bifurcation equation,

$$
\frac{d y_{11}}{d t}=\beta_{11} y_{11}+\alpha y_{11}^{3}+o\left(\left|y_{11}\right|^{3}\right) .
$$

The coefficient $\alpha \equiv \alpha(\lambda)$ is given explicitly in terms of the eigenfunctions of $L_{\lambda}$ and $L_{\lambda}^{*}$,

$$
\alpha(\lambda)=\alpha_{2}(\lambda)+\alpha_{3}(\lambda)
$$

where

$$
\begin{aligned}
& \alpha_{2}(\lambda)=\frac{2}{<w_{11}, w_{11}^{*}>} \sum_{i=1,2} \frac{\left.<G_{2}\left(w_{11}\right), w_{2 i}^{*}><G_{2}\left(w_{11}, w_{2 i}\right), w_{11}^{*}\right\rangle}{\left(2 \beta_{11}-\beta_{2 i}\right)<w_{2 i}, w_{2 i}^{*}>}, \\
& \alpha_{3}(\lambda)=\frac{1}{<w_{11}, w_{11}^{*}>}<G_{3}\left(w_{11}\right), w_{11}^{*}>.
\end{aligned}
$$

Here, $<\cdot, \cdot>$ denotes the inner product in $H$. (The subscript $\lambda$ on the $k$-linear forms has been omitted.)

Proof. We look for a solution $w$ of Eq. (2.13) of the form (4.5). In the space spanned by the eigenvector $w_{11}$, Eq. (2.13) reduces to

$$
\begin{aligned}
<w_{11}, w_{11}^{*}>\frac{d y_{11}}{d t} & =<L_{\lambda} w, w_{11}^{*}>+<G_{\lambda}(w), w_{11}^{*}> \\
& =\beta_{11}<w_{11}, w_{11}^{*}>y_{11}+\sum_{k=2}^{\infty}<G_{k}(w), w_{11}^{*}>.
\end{aligned}
$$

To evaluate the contributions from the various terms in the sum, we use the asymptotic expression for the center-manifold function near $\lambda_{0}$ given in the Appendix (Section A.1, Theorem A.1),

$$
y_{k i}=\Phi_{k i}^{\lambda}\left(y_{11}\right)=\frac{<G_{2}\left(w_{11}\right), w_{k i}^{*}>y_{11}^{2}}{\left(2 \beta_{11}-\beta_{k i}\right)<w_{k i}, w_{k i}^{*}>}+o\left(\left|y_{11}\right|^{2}\right), \quad k=2,3, \ldots
$$


The contribution from the bilinear form $(k=2)$ is

$$
\begin{aligned}
<G_{2}(w), w_{11}^{*}>=< & G_{2}\left(y_{11} w_{11}+z\right), w_{11}^{*}> \\
=< & G_{2}\left(w_{11}\right), w_{11}^{*}>y_{11}^{2} \\
& +2<G_{2}\left(w_{11}, z\right), w_{11}^{*}>y_{11}+<G_{2}(z), w_{11}^{*}>.
\end{aligned}
$$

The first term in the right member vanishes, because

$$
<G_{2}\left(w_{11}\right), w_{11}^{*}>=0 .
$$

The second and third term can be evaluated by means of the asymptotic expression (4.9) for the center manifold,

$$
\begin{aligned}
<G_{2}\left(w_{11}, z\right), w_{11}^{*}> & =\sum_{i=1,2}<G_{2}\left(w_{11}, w_{2 i}\right), w_{11}^{*}>y_{2 i}+o\left(\left|y_{11}\right|^{2}\right) \\
& =\frac{1}{2} \alpha_{2}<w_{11}, w_{11}^{*}>y_{11}^{2}+o\left(\left|y_{11}\right|^{2}\right), \\
<G_{2}(z), w_{11}^{*}> & =o\left(\left|y_{11}\right|^{3}\right),
\end{aligned}
$$

where $\alpha_{2}$ is defined in Eq. (4.7). Putting everything together, we obtain the asymptotic result

$$
<G_{2}(w), w_{11}^{*}>=\alpha_{2}<w_{11}, w_{11}^{*}>y_{11}^{3}+o\left(\left|y_{11}\right|^{3}\right) .
$$

The contribution from the trilinear form $(k=3)$ is

$$
\begin{aligned}
<G_{3}(w), w_{11}^{*}> & =<G_{3}\left(w_{11}\right), w_{11}^{*}>y_{11}^{3}+o\left(\left|y_{11}\right|^{3}\right) \\
& =\alpha_{3}<w_{11}, w_{11}^{*}>y_{11}^{3}+o\left(\left|y_{11}\right|^{3}\right),
\end{aligned}
$$

where $\alpha_{3}$ is defined in Eq. (4.7). The higher-order forms contribute only terms of $o\left(\left|y_{11}\right|^{3}\right)$.

4.2. Structure of the Bifurcated Object. The following theorem shows that the sign of a single number (namely, $\alpha\left(\lambda_{0}\right)$ ) characterizes the type of transitions that the system undergoes as the bifurcation parameter $\lambda$ crosses the critical curve $\Lambda_{1}$.

Theorem 4.2. Let $\Omega=(0, \ell), \lambda_{0} \in \Lambda_{1}$, and $\lambda$ near $\lambda_{0}$.

We distinguish two cases.

Case 1. $\alpha\left(\lambda_{0}\right)<0$. The system undergoes a continuous transition as $\lambda$ crosses $\Lambda_{1}$ from $R_{1}^{-}$into $R_{1}^{+}$. In particular,

(1) $w=0$ is a locally asymptotically stable equilibrium point of Eq. (2.13) for $\lambda \in R_{1}^{-}$and $\lambda \in \Lambda_{1}$;

(2) The solution of Eq. (2.13) bifurcates supercritically to an attractor $\mathcal{A}_{\lambda}$ as $\lambda$ crosses $\Lambda_{1}$ from $R_{1}^{-}$into $R_{1}^{+}$; 
(3) $\mathcal{A}_{\lambda}$ consists of two steady-state points, $w_{\lambda}^{+}$and $w_{\lambda}^{-}$,

$$
w_{\lambda}^{ \pm}= \pm\left(\beta_{11} /|\alpha|\right)^{1 / 2} w_{11}+\omega_{\lambda}, \quad \lambda \in R_{1}^{+},
$$

where $\left\|\omega_{\lambda}\right\|_{H}=o\left(\beta_{11}^{1 / 2}\right)$;

(4) there exists an open set $U_{\lambda} \subset H$ with $0 \in U_{\lambda}$ such that $\mathcal{A}_{\lambda}$ attracts $U_{\lambda} \backslash \Gamma$ in $H$, where $\Gamma$ is the stable manifold of 0 with codimension 1 ; and

(5) there exists an $\varepsilon>0$ and two disjoint open sets $U_{\lambda}^{+}, U_{\lambda}^{-} \subset H$ with $0 \in \partial U_{\lambda}^{+} \cap \partial U_{\lambda}^{-}$such that $w_{\lambda}^{ \pm} \in U_{\lambda}^{ \pm}$and $\lim _{t \rightarrow \infty} \| w\left(t ; w_{0}\right)-$ $w_{\lambda}^{ \pm} \|_{H}=0$ for any solution $w\left(t ; w_{0}\right)$ of $E q$. (2.13) satisfying the initial condition $w\left(0 ; w_{0}\right)=w_{0} \in U_{\lambda}^{ \pm}$and any $\lambda$ satisfying the condition $\operatorname{dist}\left(\lambda_{0}, \lambda\right)<\varepsilon$.

Case 2. $\alpha\left(\lambda_{0}\right)>0$. The system undergoes a discontinuous (jump) transition as $\lambda$ crosses $\Lambda_{1}$ from $R_{1}^{-}$into $R_{1}^{+}$. In particular,

(1) the solution of Eq. (2.13) bifurcates subcritically from $\left(\lambda_{0}, 0\right)$ to a repeller $\mathcal{R}_{\lambda}$ as $\lambda$ crosses $\Lambda_{1}$ from $R_{1}^{+}$into $R_{1}^{-}$; and

(2) $\mathcal{R}_{\lambda}$ consists of two steady-state points, $w_{\lambda}^{+}$and $w_{\lambda}^{-}$,

$$
w_{\lambda}^{ \pm}= \pm\left(\left|\beta_{11}\right| / \alpha\right)^{1 / 2} w_{11}+\omega_{\lambda}, \quad \lambda \in R_{1}^{-},
$$

where $\left\|\omega_{\lambda}\right\|_{H}=o\left(\left|\beta_{11}\right|^{1 / 2}\right) ;$

Proof. Case 1: $\alpha\left(\lambda_{0}\right)<0$.

Equation (4.6) shows that $w=0$ is a locally asymptotically stable equilibrium point.

According to the attractor-bifurcation theorem (Section A.2, Theorem A.2), the system bifurcates at $\left(\lambda_{0}, 0\right)$ to an attractor $\mathcal{A}_{\lambda}$ as $\lambda$ transits from $R_{1}^{-}$into $R_{1}^{+}$.

The structure of $\mathcal{A}_{\lambda}$ follows from the stationary form of Eq. (4.6),

$$
\beta_{11} y_{11}+\alpha y_{11}^{3}+o\left(\left|y_{11}\right|^{3}\right)=0 \text {. }
$$

The number and nature of the solutions of this equation do not change if the terms of $o\left(\left|y_{11}\right|^{3}\right)$ are ignored, provided all solutions are regular at the origin. Thus, we find two solutions near $y=0$, namely, $y_{11}=$ $\pm\left(\beta_{11} /|\alpha|\right)^{1 / 2}+o\left(\beta^{1 / 2}\right)$.

Case 2: $\alpha\left(\lambda_{0}\right)>0$.

The attractor-bifurcation theorem applies to the time-reversed form $(s=-t)$ of Eq. (4.6),

$$
\frac{d y_{11}}{d s}=-\beta_{11} y_{11}+(-\alpha) y_{11}^{3}+o\left(\left|y_{11}\right|^{3}\right) .
$$

The statements of the theorem follow by reversing time back again. 
Theorem 4.2 shows that, if $\alpha\left(\lambda_{0}\right)<0$ (Case 1 ), the attractor consists of two steady-state points, each with its own basin of attraction. The attractor bifurcation is shown schematically in Fig. 2. From the perspective of pattern formation, the theorem predicts a smooth transition to one of two types of patterns that differ only in phase; which of the two patterns is actually realized depends on the initial data.

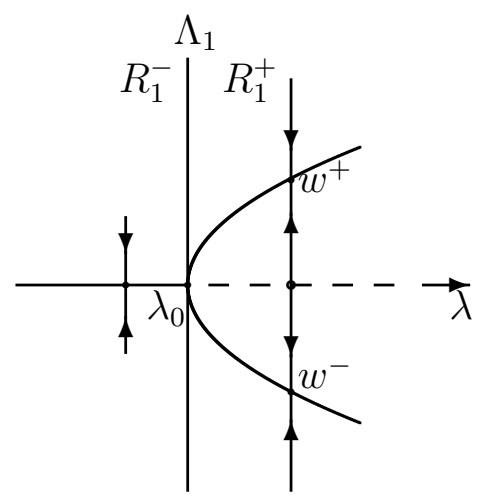

FiguRE 2. One-dimensional domain: supercritical bifurcation to an attractor $\mathcal{A}=\left\{w^{+}, w^{-}\right\}$.

The theorem shows, furthermore, that if $\alpha\left(\lambda_{0}\right)>0$ (Case 2), there is a jump transition as $\lambda$ crosses $\Lambda_{1}$ from $R_{1}^{-}$into $R_{1}^{+}$. In this case, there is a neighborhood $U$ of the basic solution such that the solution of the system moves away from $U$ for any initial data in $U \backslash \Gamma$, uniformly for all $\lambda \in R_{1}^{+}$near $\lambda_{0}$. This observation justifies the notion of jump transition. If the system possesses a global attractor, then the jump transition states are represented by a local attractor, away from the basic solution. This local attractor corresponds to the part of the global attractor that is realized after the transition into $R_{1}^{+}$. It extends to a local attractor for $\lambda \in R_{1}^{-}$that, together with the trivial solution, represents the metastable states. A similar phenomenon was encountered recently in a problem of superconductivity [11].

4.3. Center-Manifold Reduction - General Case. The previous arguments were focused on bifurcations associated with a transition of the parameter $\lambda$ across the curve $\Lambda_{1}$ (Fig. 1), when the leading eigenvalue $\beta_{11}$ changes sign. When $\lambda$ transits across one of the higherorder curves $\Lambda_{k}(k>1)$, the leading eigenvalue is not necessarily $\beta_{11}$. When $\lambda$ crosses a curve $\Lambda_{k}, \beta_{k 1}$ undergoes a sign change, while all eigenvalues $\beta_{j 1}$ with $j>k$ keep the same sign (negative below, positive above the curve). Since the multiplicity of $\beta_{k 1}$ is one, results similar to those for $k=1$ are obtained; the only difference appears in the 
value of $\alpha$. The center-manifold reduction can be performed, and the interactions between eigenvalues can be calculated as in the proof of Lemma 4.1. The reduced bifurcation equation is of a pitchfork type,

$$
\frac{d y_{k 1}}{d t}=\beta_{k 1} y_{k 1}+\alpha^{k} y_{k 1}^{3}+o\left(\left|y_{k 1}\right|^{3}\right),
$$

and the coefficient $\alpha^{k} \equiv \alpha^{k}(\lambda)$ can be found by calculating the interactions of the eigenfunctions of $L_{\lambda}$ and $L_{\lambda}^{*}$,

$$
\alpha^{k}(\lambda)=\alpha_{2}^{k}(\lambda)+\alpha_{3}^{k}(\lambda) .
$$

Since $\int_{0}^{\ell} \varphi_{k} \varphi_{k} \varphi_{m}=0$ unless $m=2 k$, the only nonzero contributions come from $m=2 k$,

$$
\begin{aligned}
& \alpha_{2}^{k}(\lambda)=\frac{2}{<w_{k 1}, w_{k 1}^{*}>} \sum_{i=1,2} \frac{<G_{2}\left(w_{k 1}\right), w_{2 k, i}^{*}><G_{2}\left(w_{k 1}, w_{2 k, i}\right), w_{k 1}^{*}>}{\left(2 \beta_{k 1}-\beta_{2 k, i}\right)<w_{2 k, i}, w_{2 k, i}^{*}>}, \\
& \alpha_{3}^{k}(\lambda)=\frac{1}{<w_{k 1}, w_{k 1}^{*}>}<G_{3}\left(w_{k 1}\right), w_{k 1}^{*}>
\end{aligned}
$$

\section{Bifurcation Analysis - Two-Dimensional Domains}

Let $\Omega=\left(0, \ell_{1}\right) \times\left(0, \ell_{2}\right)$. As in the one-dimensional case, we reduce Eq. (2.13) to its center-manifold representation near $\lambda_{0}$.

The eigenvalues and eigenvectors of the negative Laplacian subject to Neumann boundary conditions (Eqs. (4.3) and (4.4)) are

$$
\begin{aligned}
\rho_{k_{1} k_{2}} & =\rho_{k_{1}}+\rho_{k_{2}}, \quad \rho_{k_{i}}=k_{i}^{2}\left(\pi / \ell_{i}\right)^{2}, i=1,2 \\
\varphi_{k_{1} k_{2}}(x) & =\cos \left(x_{1} \sqrt{ } \rho_{k_{1}}\right) \cos \left(x_{2} \sqrt{ } \rho_{k_{2}}\right), x=\left(x_{1}, x_{2}\right) \in \Omega .
\end{aligned}
$$

Here, $k_{1}$ and $k_{2}$ range over all nonnegative integers such that $|k|=$ $k_{1}+k_{2}=1,2, \ldots$.

The eigenvalues $\beta_{k_{1} k_{2} i}(i=1,2)$ and the corresponding eigenvectors of $L_{\lambda}$ are given in Eqs. (3.5) and (3.6), respectively, where $k$ now stands for the ordered pair $\left(k_{1}, k_{2}\right)$.

The dynamics depend on the relative size of $\ell_{1}$ and $\ell_{2}$. On a rectangular (nonsquare) domain, the dynamics are essentially the same as on a one-dimensional domain. For example, if $\ell_{2}<\ell_{1}$, then $\rho_{10}=\left(\pi / \ell_{1}\right)^{2}$ is the smallest eigenvalue of the negative Laplacian, with corresponding eigenvector $\varphi_{10}=\cos \left(x_{1} \sqrt{ } \rho_{1}\right)$, and the leading eigenvalue of $L_{\lambda}$ is $\beta_{101}$. This eigenvalue is simple, and the corresponding eigenvector is

$$
w_{101}=\left(\begin{array}{c}
-\gamma f_{v} \cos \left(x_{1} \sqrt{ } \rho_{1}\right) \\
\left(\gamma f_{u}-\rho_{1}-\beta_{101}\right) \cos \left(x_{1} \sqrt{ } \rho_{1}\right)
\end{array}\right) .
$$


The center-manifold reduction leads to a one-dimensional dynamical system similar to Eq. (4.6). Lemma 4.1 and Theorem 4.2 apply verbatim if $\beta_{11}$ is replaced by $\beta_{101}$ and $w_{11}$ by $w_{101}$ everywhere.

On the other hand, the dynamics become qualitatively different if the domain is square - that is, if $\ell_{1}=\ell_{2}=\ell$ and $\Omega=(0, \ell)^{2}$. Then $\rho_{k_{1} k_{2}}=\rho_{k_{2} k_{1}}$ for any pair $\left(k_{1}, k_{2}\right)$, so $\beta_{k_{1} k_{2} i}=\beta_{k_{2} k_{1} i}(i=1,2)$. To avoid notational complications, we consider two eigenvalues as distinct, even if they coincide because of symmetry, and associate each with its own eigenvector. Thus, we associate the eigenvector

$$
w_{k_{1} k_{2} i}=\left(\begin{array}{c}
-\gamma f_{v} \varphi_{k_{1} k_{2}} \\
\left(\gamma f_{u}-\rho_{k_{1} k_{2}}-\beta_{k_{1} k_{2} i}\right) \varphi_{k_{1} k_{2}}
\end{array}\right)
$$

with the eigenvalue $\beta_{k_{1} k_{2} i}$ and the eigenvector

$$
w_{k_{1} k_{2} i}^{*}=\left(\begin{array}{c}
-\gamma g_{u} \varphi_{k_{1} k_{2}} \\
\left(\gamma f_{u}-\rho_{k_{1} k_{2}}-\beta_{k_{1} k_{2} i}^{*}\right) \varphi_{k_{1} k_{2}}
\end{array}\right)
$$

with the eigenvalue $\beta_{k_{1} k_{2} i}^{*}$, whether $k_{1}$ and $k_{2}$ are equal or not.

5.1. Center-Manifold Reduction. The leading eigenvalues are $\beta_{101}$ and $\beta_{011}$. These eigenvalues coincide, but we consider them separately, each with its own eigenvector. The two eigenvalues pass (together) through 0 as $\lambda$ crosses $\Lambda_{1}$ at $\lambda=\lambda_{0}$.

Theorem 5.1. Near $\lambda_{0} \in \Lambda_{1}$, the solution of Eq. (2.13) can be expressed in the form

$$
w=y_{1} w_{1}+y_{2} w_{2}+z, \quad z=\sum_{\left(k_{1}, k_{2}\right):|k|=2,3, \ldots} \sum_{i=1,2} y_{k_{1} k_{2} i} w_{k_{1} k_{2} i}
$$

where $w_{1}=w_{101}$ and $w_{2}=w_{011}$. The coefficients $y_{1}$ and $y_{2}$ of the leading terms satisfy a system of equations of the form

$$
\begin{aligned}
& \frac{d y_{1}}{d t}=\beta_{101} y_{1}+\left(\alpha y_{1}^{2}+\sigma y_{2}^{2}\right) y_{1}+o\left(\left|y_{1}\right|^{3}+\left|y_{2}\right|^{3}\right), \\
& \frac{d y_{2}}{d t}=\beta_{011} y_{2}+\left(\alpha y_{2}^{2}+\sigma y_{1}^{2}\right) y_{2}+o\left(\left|y_{1}\right|^{3}+\left|y_{2}\right|^{3}\right),
\end{aligned}
$$

where $\beta_{101}=\beta_{011}$. The coefficients $\alpha \equiv \alpha(\lambda)$ and $\sigma \equiv \sigma(\lambda)$ are given explicitly in terms of the eigenfunctions of $L_{\lambda}$ and $L_{\lambda}^{*}$,

$$
\alpha(\lambda)=\underset{15}{\alpha_{2}(\lambda)}+\alpha_{3}(\lambda)
$$


where

$$
\begin{aligned}
& \alpha_{2}(\lambda)=\frac{2}{<w_{1}, w_{1}^{*}>} \sum_{i=1,2} \frac{<G_{2}\left(w_{1}\right), w_{20 i}^{*}><G_{2}\left(w_{1}, w_{20 i}\right), w_{1}^{*}>}{\left(2 \beta_{101}-\beta_{20 i}\right)<w_{20 i}, w_{20 i}^{*}>}, \\
& \alpha_{3}(\lambda)=\frac{1}{<w_{1}, w_{1}^{*}>}<G_{3}\left(w_{1}\right), w_{1}^{*}>,
\end{aligned}
$$

and

$$
\sigma(\lambda)=\sigma_{2}(\lambda)+\sigma_{3}(\lambda)
$$

where

$$
\begin{aligned}
& \sigma_{2}(\lambda)=\frac{4}{<w_{1}, w_{1}^{*}>} \sum_{i=1,2} \frac{<G_{2}\left(w_{1}, w_{2}\right), w_{11}^{*}><G_{2}\left(w_{2}, w_{11 i}\right), w_{1}^{*}>}{\left(2 \beta_{101}-\beta_{11 i}\right)<w_{11 i}, w_{11 i}^{*}>}, \\
& \sigma_{3}(\lambda)=\frac{3}{<w_{1}, w_{1}^{*}>}<G_{3}\left(w_{1}, w_{2}, w_{2}\right), w_{1}^{*}>.
\end{aligned}
$$

Proof. We look for a solution $w$ of Eq. (2.13) of the form (5.4). In the space spanned by the eigenvectors $w_{1}=w_{101}$ and $w_{2}=w_{011}$, Eq. (2.13) reduces to

$$
\begin{aligned}
& <w_{1}, w_{1}^{*}>\frac{d y_{1}}{d t}=\beta_{101}<w_{1}, w_{1}^{*}>y_{1}+\sum_{k=2}^{\infty}<G_{k}(w), w_{1}^{*}>, \\
& <w_{2}, w_{2}^{*}>\frac{d y_{2}}{d t}=\beta_{011}<w_{2}, w_{2}^{*}>y_{2}+\sum_{k=2}^{\infty}<G_{k}(w), w_{2}^{*}>.
\end{aligned}
$$

To evaluate the contributions from the various terms in the sums, we again use the asymptotic expression for the center-manifold function near $\lambda_{0}$ given in the Appendix (Section A.1), Theorem A.1,

$$
\begin{aligned}
y_{k_{1} k_{2} i} & =\Phi_{k_{1} k_{2} i}^{\lambda}\left(y_{1}, y_{2}\right) \\
& =\frac{\sum_{j=1,2}<G_{2}\left(w_{j}\right), w_{k_{1} k_{2} i}^{*}>y_{j}^{2}}{\left(2 \beta_{101}-\beta_{k_{1} k_{2} i}\right)<w_{k_{1} k_{2} i}, w_{k_{1} k_{2} i}^{*}>}+o\left(|y|^{2}\right), \quad k_{1} \neq k_{2}, \\
y_{k k i} & =\Phi_{k k i}^{\lambda}\left(y_{1}, y_{2}\right)=\frac{2<G_{2}\left(w_{1}, w_{2}\right), w_{k k i}^{*}>y_{1} y_{2}}{\left(2 \beta_{101}-\beta_{k k i}\right)<w_{k k i}, w_{k k i}^{*}>}+o\left(|y|^{2}\right),
\end{aligned}
$$

where $|y|^{2}=\left|y_{1}\right|^{2}+\left|y_{2}\right|^{2}$.

Consider the first of Eqs. (5.8). The contribution from the bilinear form is

$$
\begin{aligned}
<G_{2}(w), w_{1}^{*}>= & \sum_{i=1,2}<G_{2}\left(w_{i}\right), w_{1}^{*}>y_{i}^{2} \\
& +2 \sum_{i=1,2}<G_{2}\left(w_{i}, z\right), w_{1}^{*}>y_{i}+<G_{2}(z), w_{1}^{*}>.
\end{aligned}
$$


The first term in the right member vanishes, because

$$
<G_{2}\left(w_{i}\right), w_{1}^{*}>=0, \quad i=1,2 .
$$

The last term is asymptotically small,

$$
<G_{2}(z), w_{1}^{*}>=o\left(|y|^{3}\right) .
$$

The second term involves an infinite sum over $\left(k_{1}, k_{2}\right)$ with $|k|=$ $2,3, \ldots$. Many of the coefficients are zero, because of the specific form of $w_{1}, w_{2}$, and $w_{k_{1} k_{2} j}$. The nonzero terms can be evaluated asymptotically by means of the expression (5.9). In fact, the only terms that are nonzero and contribute to the leading-order (cubic) terms in $y$ are those with $i=1$ and either $\left(k_{1}, k_{2}\right)=(2,0)$ or $\left(k_{1}, k_{2}\right)=(1,1)$. Asymptotic expressions for $y_{20 i}$ and $y_{11 i}(i=1,2)$ are given in Eq. (5.9), where only the term with $j=1$ contributes to $y_{20 i}$.

Taken together, these observations show that the contribution from the bilinear form is

$$
<G_{2}(w), w_{1}^{*}>=\frac{1}{2}<w_{1}, w_{1}^{*}>\left(\alpha_{2} y_{1}^{2}+\sigma_{2} y_{1} y_{2}\right) y_{1}+o\left(|y|^{3}\right),
$$

where $\alpha_{2}$ and $\sigma_{2}$ are defined in Eqs. (5.6) and (5.7), respectively.

The contribution from the trilinear form is

$$
\begin{aligned}
<G_{3}(w), w_{1}^{*}> & =\sum_{i=1,2}<G_{3}\left(w_{i}\right), w_{1}^{*}>y_{i}^{3}+o\left(|y|^{3}\right) \\
& =<w_{1}, w_{1}^{*}>\left(\alpha_{3} y_{1}^{2}+\sigma_{3} y_{2}^{2}\right) y_{1}+o\left(|y|^{3}\right),
\end{aligned}
$$

where $\alpha_{3}$ and $\sigma_{3}$ are defined in Eq. (5.6) and (5.7), respectively.

The computations for the second of Eqs. (5.8) are similar. One finds the differential equation for $y_{2}$ given in the statement of the lemma with the same expressions for the coefficients $\alpha$ and $\sigma$. We omit the details.

5.2. Structure of the Bifurcated Object. Before analyzing the structure of the bifurcated object, we recall the following result, the proof of which can be found in Ref. [10].

Lemma 5.1. Let $y_{\lambda} \in \mathbf{R}^{2}$ be a solution of the evolution equation

$$
\frac{d y}{d t}=\lambda y-G_{\lambda, k}(y)+o\left(|y|^{k}\right),
$$

where $G_{\lambda, k}$ is a symmetric $k$-linear field with $k$ odd and $k \geq 3$ satisfying the inequalities

$$
C_{1}|y|^{k+1} \leq<G_{\lambda, k}(y), y>\leq C_{2}|y|^{k+1}
$$

for some constants $C_{2}>C_{1}>0$, uniformly in $\lambda$. Then $y_{\lambda}$ bifurcates from $(y, \lambda)=(0,0)$ to an attractor $\mathcal{A}_{\lambda}$ that is homeomorphic to $S^{1}$. 
Moreover, (i) $\mathcal{A}_{\lambda}$ is a periodic orbit, or (ii) $\mathcal{A}_{\lambda}$ consists of an infinite number of steady-state points, or (iii) $\mathcal{A}_{\lambda}$ contains at most $2(k+1)$ steady-state points. In case (iii), the steady-state points are saddle points, (possibly degenerate) stable nodes, or steady-state points with index zero; the number of saddle points equals the number of stable nodes, and both numbers are even; if there are no saddle points or stable nodes, then there is at least one steady-state point with index zero.

The following theorem shows that, in the case of diffusion on a square domain, the types of transitions that the system undergoes as the bifurcation parameter $\lambda$ crosses the critical curve $\Lambda_{1}$ are determined by two numbers, $\alpha\left(\lambda_{0}\right)$ and $\sigma\left(\lambda_{0}\right)$.

Theorem 5.2. $\Omega=(0, \ell)^{2}, \lambda_{0} \in \Lambda_{1}, \lambda$ near $\lambda_{0}$.

Case 1. $\alpha\left(\lambda_{0}\right)<0$. The system undergoes a continuous transition as $\lambda$ crosses $\Lambda_{1}$ from $R_{1}^{-}$into $R_{1}^{+}$.

- If $\sigma\left(\lambda_{0}\right)<\left|\alpha\left(\lambda_{0}\right)\right|$, then

(1) $w=0$ is a locally asymptotically stable equilibrium point of Eq. (2.13) for $\lambda \in R_{1}^{-}$and $\lambda \in \Lambda_{1}$;

(2) the solution of Eq. (2.13) bifurcates to an attractor $\mathcal{A}_{\lambda}$ as $\lambda$ crosses $\Lambda_{1}$ from $R_{1}^{-}$into $R_{1}^{+}$; and

(3) $\mathcal{A}_{\lambda}$ is homeomorphic to $S^{1}$.

- If $\sigma\left(\lambda_{0}\right)<0, \mathcal{A}_{\lambda}$ consists of an infinite number of steady-state points.

- If $0 \leq \sigma\left(\lambda_{0}\right)<\left|\alpha\left(\lambda_{0}\right)\right|, \mathcal{A}_{\lambda}$ contains eight steady-state points, which can be expressed as

$$
w_{\lambda}=W_{\lambda}+w_{\lambda}, \quad \lambda \in R_{1}^{+},
$$

where $W_{\lambda}$ belongs to the eigenspace corresponding to $\beta_{101}$ and $\left\|\omega_{\lambda}\right\|_{H}=o\left(\left\|W_{\lambda}\right\|_{H}\right)$.

Case 2. $\alpha\left(\lambda_{0}\right)>0$. The system undergoes a discontinuous (jump) transition as $\lambda$ crosses $\Lambda_{1}$ from $R_{1}^{-}$into $R_{1}^{+}$:

- If $\sigma\left(\lambda_{0}\right)>-\alpha\left(\lambda_{0}\right)$, then

(1) the solution of Eq. (2.13) bifurcates subcritically to a repeller $\mathcal{R}_{\lambda}$ as $\lambda$ crosses $\Lambda_{1}$ from $R_{1}^{+}$into $R_{1}^{-}$; and

(2) $\mathcal{R}_{\lambda}$ is homeomorphic to $S^{1}$.

- If $\sigma\left(\lambda_{0}\right)>0, \mathcal{R}_{\lambda}$ consists of an infinite number of steady-state points.

- If $-\alpha\left(\lambda_{0}\right)<\sigma\left(\lambda_{0}\right) \leq 0, \mathcal{R}_{\lambda}$ contains eight steady-state points, which can be expressed as

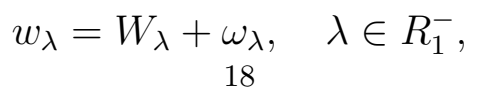


where $W_{\lambda}$ belongs to the eigenspace corresponding to $\beta_{101}$ and $\left\|\omega_{\lambda}\right\|_{H}=o\left(\left\|W_{\lambda}\right\|_{H}\right)$.

Proof. Case 1: $\alpha\left(\lambda_{0}\right)<0$,

If $\sigma\left(\lambda_{0}\right)<\left|\alpha\left(\lambda_{0}\right)\right|$, then Eq. (5.5) shows that $w=0$ is a locally asymptotically stable equilibrium point.

It follows from Lemma 5.1 and the attractor-bifurcation theorem (Section A.2, Theorem A.2) that the system bifurcates to an attractor $\mathcal{A}_{\lambda}$ as $\lambda$ transits from $R_{1}^{-}$into $R_{1}^{+}$and that $\mathcal{A}_{\lambda}$ is homeomorphic to $S^{1}$.

The structure of the bifurcated attractor is found from the stationary form of Eq. (5.5). Ignoring the terms of $o\left(|y|^{3}\right)$, we have the system of equations

$$
\begin{aligned}
& \left(\beta_{101}+\alpha y_{1}^{2}+\sigma y_{2}^{2}\right) y_{1}=0 \\
& \left(\beta_{011}+\alpha y_{2}^{2}+\sigma y_{1}^{2}\right) y_{2}=0
\end{aligned}
$$

where $\beta_{101}=\beta_{011}$.

If $\sigma\left(\lambda_{0}\right)<0$, the system (5.14) admits an infinite number of solutions near $y=0$. If $\alpha\left(\lambda_{0}\right)<0$ and $0 \leq \sigma\left(\lambda_{0}\right)<\left|\alpha\left(\lambda_{0}\right)\right|$, it admits eight nonzero solutions near $y=0$,

$$
\begin{aligned}
& y_{1}=0, \quad y_{2}^{2}=\beta_{101} /|\alpha| ; \\
& y_{2}=0, \quad y_{1}^{2}=\beta_{101} /|\alpha| ; \\
& y_{1}^{2}=y_{2}^{2}=\beta_{101} /|\alpha+\sigma|
\end{aligned}
$$

These solutions are regular, so Eq. (5.5) also has the same number of steady-state solutions; they differ from the solutions of Eq. (5.14) by terms that are $o(|y|)$.

Case 2: $\alpha\left(\lambda_{0}\right)>0$.

The attractor-bifurcation theorem applies to the time-reversed form $(s=-t)$ of Eq. (5.5),

$$
\begin{aligned}
& \frac{d y_{1}}{d s}=-\beta_{101} y_{1}+\left(-\alpha y_{1}^{2}-\sigma y_{2}^{2}\right) y_{1}+o\left(\left|y_{1}\right|^{3}+\left|y_{2}\right|^{3}\right), \\
& \frac{d y_{2}}{d t}=-\beta_{011} y_{2}+\left(-\alpha y_{2}^{2}-\sigma y_{1}^{2}\right) y_{2}+o\left(\left|y_{1}\right|^{3}+\left|y_{2}\right|^{3}\right),
\end{aligned}
$$

The statements of the theorem follow by reversing time back again.

Theorem 5.2 shows that, if $\alpha\left(\lambda_{0}\right)<0$ and $\sigma\left(\lambda_{0}\right)<\left|\alpha\left(\lambda_{0}\right)\right|$ (Case 1), the bifurcation is an $S^{1}$-attractor bifurcation. If both $\alpha\left(\lambda_{0}\right)$ and $\sigma\left(\lambda_{0}\right)$ are negative, the attractor consists of an infinite number of steady-state points; on the other hand, if $\alpha\left(\lambda_{0}\right)<0$ and $0 \leq \sigma\left(\lambda_{0}\right)<\left|\alpha\left(\lambda_{0}\right)\right|$, the attractor contains eight steady-state points. Figure 3 , for instance, shows the phase diagram on the center manifold after bifurcation, where eight 
steady states are connected by heteroclinic orbits. The odd-indexed points $\left(P_{1}, P_{3}, P_{5}\right.$, and $\left.P_{7}\right)$ are minimal attractors; the even-indexed points $\left(P_{2}, P_{4}, P_{6}\right.$, and $\left.P_{8}\right)$ are saddle points.

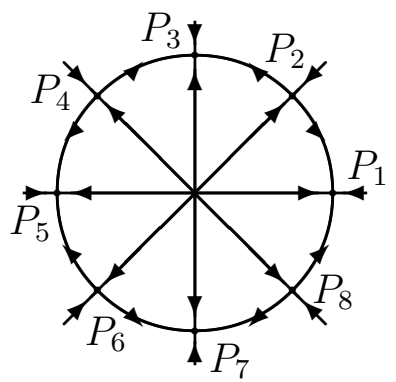

Figure 3. Two-dimensional domain: $S^{1}$-bifurcation with eight regular steady states.

5.3. Square Domain - General Case. As mentioned in Section 4.3, the leading eigenvalue may change as one passes from one curve $\Lambda_{k}$ to the next. In a one-dimensional domain the leading eigenvalue remains simple, but in a two-dimensional domain this need no longer be the case. For instance, the multiplicity of any eigenvalue $\beta_{k_{1} k_{2} i}$ on a square domain is two if $k_{1} \neq k_{2}$, one if $k_{1}=k_{2}$.

If $k_{1}=k_{2}, \beta_{111}$ is the leading eigenvalue, and the center-manifold reduction leads to a reduced dynamical equation of cubic type,

$$
\frac{d y}{d t}=\beta_{111} y+\alpha y^{3}+o\left(|y|^{3}\right)
$$

where the coefficient $\alpha \equiv \alpha(\lambda)$ can be expressed in terms of the interaction between eigenfunctions of $L_{\lambda}$ and $L_{\lambda}^{*}$. Therefore, Theorems 4.2 applies verbatim if $\beta_{11}$ is replaced by $\beta_{111}, w_{11}$ by $w_{111}$, and, more important, $\alpha$ by

$$
\alpha(\lambda)=\alpha_{2}(\lambda)+\alpha_{3}(\lambda)
$$

where

$$
\begin{aligned}
& \alpha_{2}(\lambda)=\frac{2}{<w_{111}, w_{111}^{*}>} \\
& \times \sum_{i=1,2} \sum_{j=1,2} \frac{<G_{2}\left(w_{111}\right), w_{20 i j}^{*}><G_{2}\left(w_{111}, w_{20 i j}\right), w_{111}^{*}>}{\left(2 \beta_{111}-\beta_{20 i}\right)<w_{20 i j}, w_{20 i j}^{*}>} \\
& \alpha_{3}(\lambda)=\frac{1}{<w_{111}, w_{111}^{*}>}<G_{3}\left(w_{111}\right), w_{111}^{*}> \\
& 20
\end{aligned}
$$


In fact, the projection procedure involves the calculation of a few more terms. Writing the solution $w$ of Eq. (2.13) in the form

$$
w=y w_{111}+z, \quad z=\sum_{\left(k_{1}, k_{2}\right) \neq(1,1)} \sum_{i=1,2} y_{k_{1} k_{2} i} w_{k_{1} k_{2} i}
$$

and projecting in the direction of the first eigenvalue, we obtain the equation

$$
<w_{111}, w_{111}^{*}>\frac{d y}{d t}=\beta_{111}<w_{111}, w_{111}^{*}>y+\sum_{k=2}^{\infty}<G_{k}(w), w_{111}^{*}>.
$$

Using again the center-manifold function near $\lambda_{0}$ to evaluate the contributions from the various terms in the sums, we obtain

$$
y_{20 i j}=\Phi_{20 i j}^{\lambda}(y)=\frac{<G_{2}(w), w_{20 i j}^{*}>y^{2}}{\left(2 \beta_{111}-\beta_{20 i}\right)<w_{20 i j}, w_{20 i j}^{*}>}+o\left(|y|^{2}\right) .
$$

The contribution from the bilinear form is

$$
\begin{aligned}
<G_{2}(w), w_{111}^{*}>=< & G_{2}\left(w_{111}\right), w_{111}^{*}>y^{2}+<G_{2}(z), w_{111}^{*}> \\
& +2<G_{2}\left(w_{111}, z\right), w_{111}^{*}>y .
\end{aligned}
$$

A straightforward computation shows that

$$
<G_{2}\left(w_{111}\right), w_{111}^{*}>=0 .
$$

Using Eq. (5.20), we obtain

$$
\begin{aligned}
<G_{2}(z), w_{111}^{*}> & =o\left(|y|^{3}\right), \\
<G_{2}\left(w_{111}, z\right), w_{111}^{*}>= & \sum_{i=1,2} \sum_{j=1,2}<G_{2}\left(w_{111}, w_{20 i j}\right), w_{111}^{*}>y_{20 i j} \\
& +o\left(|y|^{2}\right) .
\end{aligned}
$$

Thus,

$$
<G_{2}(w), w_{1}^{*}>=\alpha_{2}<w_{111}, w_{111}^{*}>y^{3}+o\left(|y|^{3}\right),
$$

where $\alpha_{2}$ is defined in Eq. (5.18).

The contribution from the trilinear form $(k=3)$ is

$$
\begin{aligned}
<G_{3}(w), w_{111}^{*}> & =<G_{3}\left(w_{111}\right), w_{111}^{*}>y^{3}+o\left(|y|^{3}\right) \\
& =\alpha_{3}<w_{111}, w_{111}^{*}>y^{3}+o\left(|y|^{3}\right),
\end{aligned}
$$

where $\alpha_{3}$ is defined in Eq. (5.18).

When $k_{1} \neq k_{2}$, the multiplicity of the eigenvalue is two, and the center-manifold reduction yields two equations. If $\beta_{k 0}$ is the leading eigenvalue and $\beta_{k 0}$ changes sign at a critical point, the reduction procedure yields two equations similar to Eqs. (5.5). 
Remark 1. The center-manifold reduction simplifies considerably when the original system has certain spatial or other symmetries. An indication of such a simplification can be gleaned, for example, from Eq. (5.1), where the two equations have identical coefficients. However, the method is essentially independent of any symmetry.

Remark 2. The results of Sections 4 and 5 remain valid if the Neumann (zero-flux) boundary conditions are replaced by periodic boundary conditions. In fact, the form of the reduced center-manifold equations and the expressions for the parameters $\alpha$ and $\sigma$ remain the same; only the eigenvalues and eigenvectors of the negative Laplacian are different.

\section{Example - Schnakenberg Equation}

We illustrate the preceding results on a classical model for pattern formation in complex biological structures due to Schnakenberg [17],

$$
\begin{aligned}
U_{t} & =\Delta U+\gamma\left(a-U+U^{2} V\right), \\
V_{t} & =d \Delta V+\gamma\left(b-U^{2} V\right) .
\end{aligned}
$$

The constants $a$ and $b$ are positive; $\lambda=(\gamma, d)$ is the bifurcation parameter. The system admits a uniform steady state $(\bar{u}, \bar{v})$,

$$
\bar{u}=a+b, \quad \bar{v}=\frac{b}{(a+b)^{2}} .
$$

If $U=\bar{u}+u$ and $V=\bar{v}+v$, then $u$ and $v$ must satisfy the equations

$$
\begin{aligned}
& u_{t}=\Delta U+\gamma\left(\frac{b-a}{a+b} u+(a+b)^{2} v+\frac{b}{(a+b)^{2}} u^{2}+2(a+b) u v+u^{2} v\right), \\
& v_{t}=d \Delta V-\gamma\left(\frac{2 b}{a+b} u+(a+b)^{2} v+\frac{b}{(a+b)^{2}} u^{2}+2(a+b) u v+u^{2} v\right) .
\end{aligned}
$$

This system of equations is of the type (2.13), with

$$
B=\left(\begin{array}{cc}
\frac{b-a}{a+b} & (a+b)^{2} \\
-\frac{2 b}{a+b} & -(a+b)^{2}
\end{array}\right) .
$$

The nonlinear terms

$$
f_{1}(u, v)=-g_{1}(u, v)=\frac{b}{(a+b)^{2}} u^{2}+2(a+b) u v+u^{2} v
$$


correspond to the bilinear and trilinear forms

$$
\begin{aligned}
G_{2}(\xi, \eta) & =\gamma\left(\frac{b}{(a+b)^{2}} \xi_{1} \eta_{1}+(a+b)\left(\xi_{1} \eta_{2}+\xi_{2} \eta_{1}\right)\right)\left(\begin{array}{c}
1 \\
-1
\end{array}\right), \\
G_{3}(\xi, \eta, \zeta) & =\frac{1}{3} \gamma\left(\xi_{1} \eta_{1} \zeta_{2}+\xi_{1} \eta_{2} \zeta_{1}+\xi_{2} \eta_{1} \zeta_{1}\right)\left(\begin{array}{c}
1 \\
-1
\end{array}\right),
\end{aligned}
$$

for any vectors $\xi=\left(\begin{array}{l}\xi_{1} \\ \xi_{2}\end{array}\right), \eta=\left(\begin{array}{l}\eta_{1} \\ \eta_{2}\end{array}\right), \zeta=\left(\begin{array}{l}\zeta_{1} \\ \zeta_{2}\end{array}\right)$ in $H$.

Note that $\operatorname{det}(B)=(a+b)^{2}$ and $\operatorname{tr}(B)=\frac{b-a}{a+b}-(a+b)^{2}$. The conditions (2.6) and (2.7) are satisfied if $0<b-a<(a+b)^{3}$. Furthermore,

$$
\begin{aligned}
\operatorname{det}\left(E_{k}(\lambda)\right) & =\gamma\left(\gamma+\rho_{k}\right)(a+b)^{2}-\rho_{k} d\left(\gamma \frac{b-a}{a+b}-\rho_{k}\right), \\
\operatorname{tr}\left(E_{k}(\lambda)\right. & =\gamma\left(\frac{b-a}{a+b}-(a+b)^{2}\right)-\rho_{k}(1+d),
\end{aligned}
$$

where $\rho_{k}, k=1,2, \ldots$ are the eigenvalues of $-\Delta$ on the domain $\Omega$ with Neumann conditions on $\partial \Omega$. The expressions for the eigenvalues $\beta_{k i}$ $(i=1,2)$ of $L_{\lambda}$ follow from Eq. (3.5); the corresponding eigenvalues $\beta_{k i}^{*}$ of $L_{\lambda}^{*}$ follow from the identities $\beta_{k 1}^{*}=\beta_{k 2}$ and $\beta_{k 2}^{*}=\beta_{k 1}$.

The curves $\Lambda_{k}$ are given by the expression

$$
d_{k}(\gamma)=\frac{\gamma\left(\gamma+\rho_{k}\right)(a+b)^{2}}{\rho_{k}\left(\gamma \frac{b-a}{a+b}-\rho_{k}\right)}, \quad \gamma>\gamma_{k}=\frac{a+b}{b-a} \rho_{k}, \quad k=1,2, \ldots
$$

The curves $\Lambda_{k}$ and $\Lambda_{k+1}$ intersect at

$$
\gamma=\gamma_{k, k+1}=\frac{1}{2}\left(\gamma_{k}+\gamma_{k+1}\right)+\left(\frac{1}{4}\left(\gamma_{k}+\gamma_{k+1}\right)^{2}+\gamma_{k} \gamma_{k+1} \frac{b-a}{a+b}\right)^{1 / 2} .
$$

6.1. One-Dimensional Case. Let $\Omega=(0, \ell)$. The eigenvalues of $-\Delta$ on $(0, \ell)$ satisfying Neumann conditions at 0 and $\ell$ are $\rho_{k}=k^{2}(\pi / \ell)^{2}$, $k=1,2, \ldots$ The eigenvectors of $L_{\lambda}$ and $L_{\lambda}^{*}$ corresponding to the eigenvalues $\beta_{k i}$ and $\beta_{k i}^{*}$ are

$$
\begin{array}{ll}
w_{k i}=\left(\begin{array}{c}
-\gamma(a+b)^{2} \cos \left(x \sqrt{ } \rho_{k}\right) \\
\left(\gamma \frac{b-a}{a+b}-\rho_{k}-\beta_{k i}\right) \cos \left(x \sqrt{ } \rho_{k}\right)
\end{array}\right), & i=1,2, \\
w_{k i}^{*}=\left(\begin{array}{c}
\gamma \frac{2 b}{a b} \cos \left(x \sqrt{ } \rho_{k}\right) \\
\left(\gamma \frac{b-a}{a+b}-\rho_{k}-\beta_{k i}^{*}\right) \cos \left(x \sqrt{ } \rho_{k}\right)
\end{array}\right), \quad i=1,2 .
\end{array}
$$

Since $\int_{0}^{\ell} \cos ^{2}\left(x \sqrt{ } \rho_{k}\right) d x=\frac{1}{2} \ell$,

$$
<w_{k i}, w_{k i}^{*}>=\frac{1}{2} \ell\left(-2 \gamma^{2} b(a+b)+\left|\gamma_{a-a}^{b+b}-\rho_{k}-\beta_{k i}\right|^{2}\right), \quad i=1,2 .
$$

Now consider a transition when $\lambda$ crosses $\Lambda_{1}$ from the region below to the region above $\Lambda_{1}$ at some critical value $\lambda_{0}$. Near $\lambda_{0}$, the eigenvalues $\beta_{11}$ and $\beta_{12}$ are real. Straightforward computations yield the 
expressions

$$
\begin{aligned}
G_{2}\left(w_{11}\right) & =h_{2}\left(2\left(\rho_{1}+\beta_{11}\right)\right) \cos ^{2}\left(x \sqrt{ } \rho_{1}\right)\left(\begin{array}{c}
1 \\
-1
\end{array}\right), \\
G_{2}\left(w_{11}, w_{2 i}\right) & =h_{2}\left(\rho_{1}+\beta_{11}+\rho_{2}+\beta_{2 i}\right) \cos \left(x \sqrt{ } \rho_{1}\right) \cos \left(x \sqrt{ } \rho_{2}\right)\left(\begin{array}{c}
1 \\
-1
\end{array}\right), \\
G_{3}\left(w_{11}\right) & =h_{3}\left(\rho_{1}+\beta_{11}\right) \cos ^{3}\left(x \sqrt{ } \rho_{1}\right)\left(\begin{array}{c}
1 \\
-1
\end{array}\right),
\end{aligned}
$$

where $h_{2}$ and $h_{3}$ are linear functions of their arguments,

$$
\begin{aligned}
& h_{2}(s)=\gamma^{2}(a+b)^{2}(\gamma(2 a-b)+(a+b) s), \\
& h_{3}(s)=\gamma^{3}(a+b)^{3}(\gamma(b-a)-(a+b) s) .
\end{aligned}
$$

Since $\int_{0}^{\ell} \cos ^{4}\left(x \sqrt{ } \rho_{1}\right) d x=\frac{3}{8} \ell$ and $\int_{0}^{\ell} \cos ^{2}\left(x \sqrt{ } \rho_{1}\right) \cos \left(x \sqrt{ } \rho_{2}\right) d x=\frac{1}{4} \ell$,

$$
\begin{aligned}
<G_{2}\left(w_{11}\right), w_{21}^{*}> & =\frac{1}{4} \ell\left(\gamma+\rho_{1}+\beta_{22}\right) h_{2}\left(\rho_{1}+\beta_{11}\right), \\
<G_{2}\left(w_{11}\right), w_{22}^{*}> & =\frac{1}{4} \ell\left(\gamma+\rho_{1}+\beta_{21}\right) h_{2}\left(\rho_{1}+\beta_{11}\right), \\
<G_{2}\left(w_{11}, w_{2 i}\right), w_{11}^{*}> & =\frac{1}{4} \ell\left(\gamma+\rho_{1}+\beta_{12}\right) h_{2}\left(\rho_{1}+\beta_{11}+\rho_{2}+\beta_{2 i}\right), \\
<G_{3}\left(w_{11}\right), w_{11}^{*}> & =\frac{3}{8} \ell\left(\gamma+\rho_{1}+\beta_{12}\right) h_{3}\left(\rho_{1}+\beta_{11}\right) .
\end{aligned}
$$

These expressions can be used in Eq. (4.7) to compute the coefficient $\alpha(\lambda)$ in the reduced bifurcation equation (4.6) as $\lambda$ varies along the curve $\Lambda_{1}$.

6.2. Two-Dimensional Case, $\Omega=(0, \ell)^{2}$. The evaluation of the inner products in the expression (5.6) and (5.7) for $\sigma$ is similar,

$$
\begin{aligned}
G_{2}\left(w_{1}\right) & =h_{2}\left(2\left(\rho_{10}+\beta_{101}\right)\right) \cos ^{2}\left(x \sqrt{ } \rho_{1}\right)\left(\begin{array}{c}
1 \\
-1
\end{array}\right), \\
G_{2}\left(w_{1}, w_{2}\right) & =h_{2}\left(2\left(\rho_{10}+\beta_{101}\right)\right) \cos \left(x \sqrt{ } \rho_{1}\right) \cos \left(y \sqrt{ } \rho_{1}\right)\left(\begin{array}{c}
1 \\
-1
\end{array}\right), \\
G_{2}\left(w_{1}, w_{20 i}\right) & =h_{2}\left(\rho_{10}+\beta_{101}+\rho_{20}+\beta_{20 i}\right) \cos \left(x \sqrt{ } \rho_{1}\right) \cos \left(x \sqrt{ } \rho_{2}\right)\left(\begin{array}{c}
1 \\
-1
\end{array}\right), \\
G_{2}\left(w_{2}, w_{11 i}\right) & =h_{2}\left(\rho_{10}+\beta_{101}+\rho_{11}+\beta_{11 i}\right) \cos \left(x \sqrt{ } \rho_{1}\right) \cos ^{2}\left(y \sqrt{ } \rho_{1}\right)\left(\begin{array}{c}
1 \\
-1
\end{array}\right), \\
G_{3}\left(w_{1}\right) & =h_{3}\left(\rho_{10}+\beta_{101}\right) \cos ^{3}\left(x \sqrt{ } \rho_{1}\right)\left(\begin{array}{c}
1 \\
-1
\end{array}\right) .
\end{aligned}
$$

The functions $h_{2}$ and $h_{3}$ are the same as in the one-dimensional case, Eq. (6.6). With the identities

$$
\int_{\Omega} \cos ^{4}\left(x \sqrt{ } \rho_{1}\right) d x d y=\frac{3}{8} \ell^{2}, \int_{\Omega} \cos ^{2}\left(x \sqrt{ } \rho_{1}\right) \cos \left(x \sqrt{ } \rho_{2}\right) d x d y=\frac{1}{4} \ell^{2},
$$




$$
\int_{\Omega} \cos ^{2}\left(x \sqrt{ } \rho_{1}\right) \cos ^{2}\left(y \sqrt{ } \rho_{1}\right) d x d y=\frac{3}{16} \ell^{2},
$$

we find

$$
\begin{aligned}
<G_{2}\left(w_{1}\right), w_{201}^{*}> & =\frac{1}{4} \ell^{2}\left(\gamma+\rho_{10}+\beta_{202}\right) h_{2}\left(\rho_{10}+\beta_{101}\right), \\
<G_{2}\left(w_{1}\right), w_{202}^{*}> & =\frac{1}{4} \ell^{2}\left(\gamma+\rho_{10}+\beta_{201}\right) h_{2}\left(\rho_{10}+\beta_{101}\right), \\
<G_{2}\left(w_{1}, w_{20 i}\right), w_{1}^{*}> & =\frac{1}{4} \ell^{2}\left(\gamma+\rho_{10}+\beta_{102}\right) h_{2}\left(\rho_{10}+\beta_{101}+\rho_{20}+\beta_{20 i}\right), \\
<G_{2}\left(w_{1}, w_{11 i}\right), w_{1}^{*}> & =\frac{3}{16} \ell^{2}\left(\gamma+\rho_{10}+\beta_{102}\right) h_{2}\left(\rho_{10}+\beta_{101}+\rho_{11}+\beta_{11 i}\right), \\
<G_{3}\left(w_{1}\right), w_{1}^{*}> & =\frac{3}{8} \ell^{2}\left(\gamma+\rho_{1}+\beta_{12}\right) h_{3}\left(\rho_{1}+\beta_{11}\right) .
\end{aligned}
$$

6.3. Numerical Results. We illustrate the above analytical results with the results of numerical computations for the Schnakenberg model on the unit interval $\Omega=(0,1)$ (one-dimensional case, 1D) and on the unit square $\Omega=(0,1)^{2}$ (two-dimensional case, 2D).

The configuration of the critical curves depends on the values of the parameters $a$ and $b$. The positive branches of $\Lambda_{1}$ and $\Lambda_{2}$ are shown in Fig. 4(a) for $a=\frac{1}{3}, b=\frac{2}{3}$, and in Fig. 5(a) for $a=2, b=100$, for both the $1 \mathrm{D}$ and $2 \mathrm{D}$ cases. The curves $\Lambda_{1}$ coincide for $1 \mathrm{D}$ and $2 \mathrm{D}$, but the curves $\Lambda_{2}$ differ.


Figure 4. Schnakenberg equation; $a=\frac{1}{3}, b=\frac{2}{3}$. 

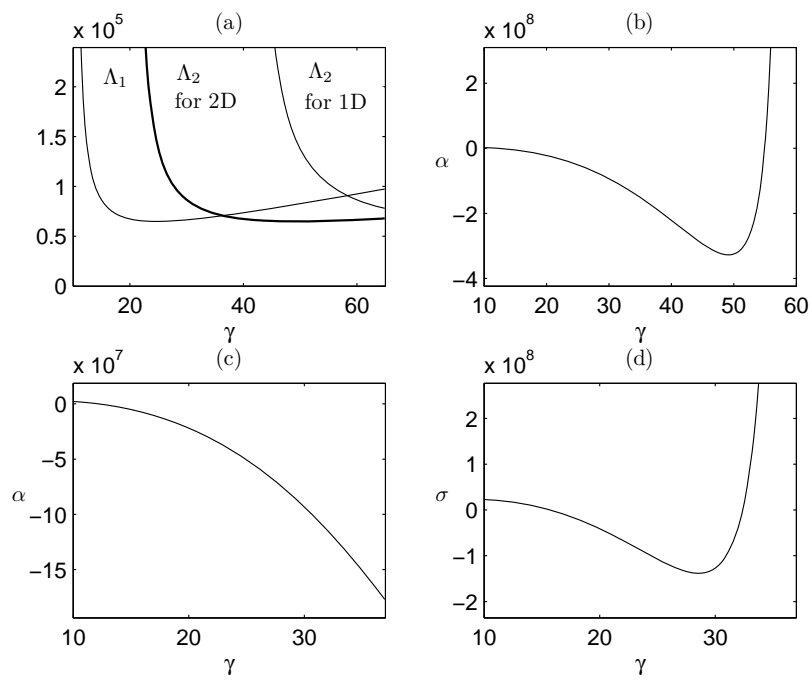

FiguRE 5. Schnakenberg equation; $a=2, b=100$.

The nature of the first critical transition is determined by the value of the bifurcation coefficient $\alpha$ in the one-dimensional case or the values of the bifurcation coefficients $\alpha$ and $\sigma$ in the two-dimensional case, at the critical value $\lambda_{0}$. The structure of the attractor and, therefore, the pattern that emerges from the bifurcation follow from Theorem 4.2 in the one-dimensional case or Theorem 5.2 in the two-dimensional case.

Consider the one-dimensional case. Figures 4(b) and 5(b) show the graph of $\alpha$ as $\lambda=\left(\gamma, d_{1}(\gamma)\right)$ moves along $\Lambda_{1}$ from the asymptote at $\gamma=\gamma_{1}=\frac{a+b}{b-a} \rho_{1}$ to the vertical line at $\gamma=\gamma_{1,2}$ where the curves $\Lambda_{1}$ and $\Lambda_{2}$ intersect. (The value of $\gamma_{1,2}$ is computed from Eq. (6.4).) When $a=\frac{1}{3}, b=\frac{2}{3}$ (Fig. 4(b)), the graph of $\alpha$ is monotonically increasing and positive, so $\alpha\left(\lambda_{0}\right)$ is always positive. The primary instability corresponds to a jump transition; the bifurcation is subcritical and results in a complex high-amplitude pattern. On the other hand, when $a=2, b=100$ (Fig. 5(b)), the graph of $\alpha$ is monotonically decreasing and negative until it reaches a minimum and turns monotonically increasing toward positive values near $\gamma_{1,2}$. Depending on the values of the constants $a$ and $b$, we can have a supercritical bifurcation (namely, when $\alpha\left(\lambda_{0}\right)<0$ ) and therefore a continuous transition resulting in a simple pattern, or a subcritical bifurcation (namely, when $\alpha\left(\lambda_{0}\right)>0$ ) and therefore a discontinuous (jump) transition resulting in a complex high-amplitude pattern.

Next consider the case of a square domain. The nature of the transition is now determined by two coefficients, $\alpha$ and $\sigma$, at the critical 
value $\lambda_{0}$. The graphs of $\alpha$ and $\sigma$ are shown in Fig. 4(c) and Fig. 4(d), respectively, for the case $a=\frac{1}{3}, b=\frac{2}{3}$ and in Fig. 5(c) and Fig. 5(d), respectively, for the case $a=2, b=100$, as $\lambda=\left(\gamma, d_{1}(\gamma)\right)$ moves along $\Lambda_{1}$ from the asymptote at $\gamma=\gamma_{1}=\frac{a+b}{b-a} \rho_{1}$ to the vertical line at $\gamma=\gamma_{1,2}$. When $a=\frac{1}{3}$ and $b=\frac{2}{3}, \alpha$ and $\sigma$ are monotonically increasing and positive. It follows from Theorem 5.2 that there exists a repeller consisting of an infinite number of steady-state points. On the other hand, when $a=2$ and $b=100, \alpha$ is monotonically decreasing and negative, while $\sigma$ is monotonically decreasing and negative until it reaches a minimum and turns monotonically increasing toward positive values near $\gamma_{1,2}$. There exists therefore an interval of critical values where $\alpha\left(\lambda_{0}\right)<0$ and $\sigma\left(\lambda_{0}\right)<0$. Hence, we see a continuous transition and a heteroclinic orbit consisting of steady-state points.

Lastly, we consider the actual dynamics of the Schnakenberg model, Eq. (2.5), and show computationally that the reduced equation obtained with the center-manifold reduction, Eq. (4.6), can be used effectively to approximate the solution of the full Schnakenberg model for values of $\lambda$ in the unstable regime. For these computations we restrict ourselves again to the unit interval. Figure 6 shows the solution $(u, v)$ of the original Schnakenberg model and the reduced equation at times $t=10$ and $t=100$. The value of $d$ was chosen so the bifurcation parameter $\lambda$ was near a critical value. In each case the difference is negligible. Since the reduced model requires substantially less computational effort than the full model, even when the latter is stiff, there is a clear advantage to using the former, especially for a qualitative evaluation.

\section{Conclusions}

In this paper we have studied the local dynamics of reaction-diffusion systems of activator-inhibitor type near a primary instability. The system is assumed to have a uniform steady state that is stable in the absence of diffusion. Diffusion introduces instabilities, and patterns emerge as a result of bifurcations from the uniform steady state. The bifurcation parameter $(\lambda)$ incorporates the ratio of the characteristic times for chemical reaction and diffusion $(\gamma)$ and the ratio of the diffusion coefficients of the competing species in the binary mixture $(d)$. For such a system, there exists a family of critical curves in the $\lambda=(\gamma, d)$ plane with the property that an exchange of stability occurs as $\lambda$ crosses one of these critical curves (Lemma 3.1). 

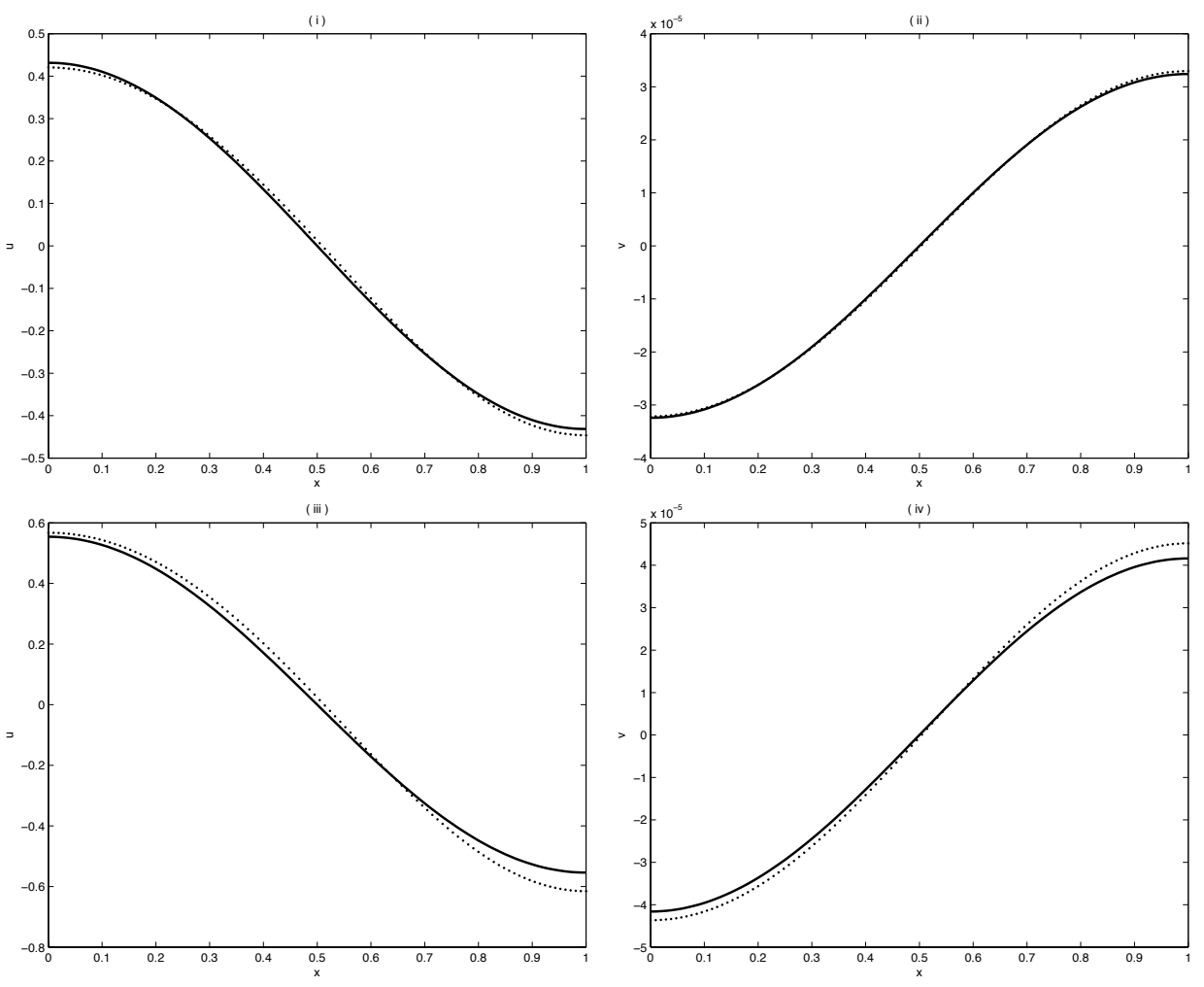

Figure 6. Solution of the Schnakenberg equation (dotted lines) and the reduced equation (solid lines) at times $t=10$ (top) and $t=100$ (bottom); $a=2, b=100$, $\gamma=55, d=87529$.

Two essential ingredients in the analysis were a new approach to the center-manifold reduction and the application of attractor-bifurcation theory. The attractor-bifurcation theory studies local invariant spheres, rather than the invariant tori of classical bifurcation theory. The center-manifold reduction method is used to derive a new set of governing equations. These equations involve some transition coefficients that are specified entirely by the eigenvalues and eigenfunctions of the underlying operators and, thus, by the physical parameters of the system, as is illustrated by the numerical results for the Schnakenberg model in Section 6.

We have shown that the bifurcated object (attractor or repeller) consists of either two points or an $S^{1}$-type object, depending on the dimension of the physical domain and the parameter regime. In the case of an $S^{1}$-bifurcation, the phase diagram after bifurcation either 
consists of an infinite number of steady-state solutions or contains eight steady states and the heteroclinic orbits connecting them.

Acknowledgments. The authors thank James Glazier (Indiana University), T. J. Kaper (Boston University), and A. Doelman (CWI, Amsterdam) for helpful discussions on the subject of pattern formation and for several of the references.

The work of H.G.K. was supported in part by the National Science Foundation under Award No. DMS-0549430-001 and by the U.S. Department of Energy under Contract No. DE-AC02-06CH11357. The work of S.W. and M.Y. was supported in part by the Office of Naval Research under contract N00014-05-1-0218 and by the National Science Foundation under contract DMS-0605067.

\section{Appendix A. Center-Manifold Reduction and Attractor BIFURCATION}

In this appendix, we summarize the center-manifold reduction and the attractor-bifurcation theory following Ref. [10]. The functional framework is that of two Hilbert spaces, $H$ and $H_{1}$, where $H_{1}$ is dense in $H$ and the inclusion $H_{1} \hookrightarrow H$ is compact.

Let $A: H_{1} \rightarrow H$ be a linear homeomorphism, and let $L_{\lambda}: H_{1} \rightarrow H$ be a compact perturbation of $A$ that depends continuously on a real parameter $\lambda$,

$$
L_{\lambda}=-A+B_{\lambda}
$$

Let $G_{\lambda}$ be a nonlinear map in $H$. Consider the initial-value problem

$$
\frac{d w}{d t}=L_{\lambda} w+G_{\lambda}(w), t>0 ; \quad w(0)=w_{0},
$$

for some given $w_{0} \in H$. We assume that $w=0$ is an equilibrium solution of Eq. (A.2) and that this solution is locally asymptotically stable.

Let $\beta_{k}(\lambda), k=1,2, \ldots$, denote the eigenvalues of $L_{\lambda}$ (counting multiplicity and ordered by their increasing real parts). Suppose that at a critical value $\lambda=\lambda_{0}$ the eigenvalues $\beta_{1}(\lambda)$ through $\beta_{m}(\lambda)$ cross the imaginary axis into the right half of the complex plane, while all eigenvalues $\beta_{k}(\lambda)$ with $k>m$ remain in the left half. Then the equilibrium solution loses stability, and a bifurcation occurs. The center-manifold reduction reduces the infinite-dimensional equation (A.2) to a finitedimensional system, and the attractor-bifurcation theorem characterizes the bifurcating solution in the neighborhood of $\lambda_{0}$. 
A.1. Center-Manifold Reduction. Decompose the space $H$ into invariant subspaces,

$$
H=E_{1} \oplus E_{2},
$$

where $E_{1}$ is the (finite-dimensional) eigenspace of $L_{\lambda}$ at $\lambda_{0}$,

$$
E_{1}=\bigcup_{k=1}^{m}\left\{w \in H_{1}:\left(L_{\lambda_{0}}-\beta_{k}\left(\lambda_{0}\right)\right)^{i} w=0, i=1,2, \ldots\right\} .
$$

Let $\widetilde{E}_{1}=E_{1}$, and define $\widetilde{E}_{2}$ as the closure of $E_{2}$ in $H$, so $H_{1}=\widetilde{E}_{1} \oplus \widetilde{E}_{2}$. The decomposition (A.3) reduces $L_{\lambda}$,

$$
L_{\lambda}=\mathcal{L}_{\lambda, 1} \oplus \mathcal{L}_{\lambda, 2}
$$

where $\mathcal{L}_{\lambda, 1}=\left.L_{\lambda}\right|_{E_{1}}: E_{1} \rightarrow \widetilde{E}_{1}$ and $\mathcal{L}_{\lambda, 2}=\left.L_{\lambda}\right|_{E_{2}}: E_{2} \rightarrow \widetilde{E}_{2}$.

The solution $w_{\lambda}$ of Eq. (A.2) can be written as

$$
w_{\lambda}=W+z, \quad W \in E_{1}, z \in E_{2},
$$

where $W$ and $z$ satisfy the system of equations

$$
\begin{aligned}
\frac{d W}{d t} & =\mathcal{L}_{\lambda, 1} W+\mathcal{G}_{\lambda, 1}(W, z), \\
\frac{d z}{d t} & =\mathcal{L}_{\lambda, 2} z+\mathcal{G}_{\lambda, 2}(W, z),
\end{aligned}
$$

with $\mathcal{G}_{\lambda, i}=P_{i} G_{\lambda}, P_{i}: H \rightarrow \widetilde{E}_{i}$ being the canonical projection.

By the classical center-manifold theorem (see, for example, Ref. [7]), there exist, for all $\lambda$ sufficiently close to $\lambda_{0}$, a neighborhood $U_{\lambda} \subset E_{1}$ of $W=0$ and a $C^{1}$ center-manifold function $\Phi^{\lambda}: U_{\lambda} \rightarrow E_{1}$ which depends continuously on $\lambda$ such that the dynamics of Eq. (A.2) are described completely by the dynamics of the finite-dimensional system

$$
\frac{d W}{d t}=\mathcal{L}_{\lambda, 1} W+\mathcal{G}_{\lambda, 1}\left(W, \Phi^{\lambda}(W)\right), \quad W \in U_{\lambda} \subset E_{1} .
$$

The following theorem gives an estimate for the center-manifold function in a neighborhood of $\lambda_{0}$.

Theorem A.1. Let $G_{\lambda}(w)=\sum_{k=p}^{\infty} G_{\lambda, k}(w)$ for some $p \geq 2$, where $G_{\lambda, k}(w) \equiv G_{\lambda, k}(w, \ldots, w)$ and $G_{\lambda, k}$ is a k-linear $C^{\infty}$ map from $H_{1} \times$ $\cdots \times H_{1}$ into $H$ for each $k$. Then

$$
\begin{gathered}
\Phi^{\lambda}(W)=\left(-\mathcal{L}_{\lambda, 2}\right)^{-1} P_{2} G_{\lambda, p}(W)+O\left(|\Re \beta(\lambda)| \cdot\|W\|^{p}\right) \\
+o\left(\|W\|^{p}\right), \quad W \in E_{1}, \quad \lambda \rightarrow \lambda_{0} .
\end{gathered}
$$

Here, $\beta$ stands for the vector of eigenvalues $\left(\beta_{1}, \ldots, \beta_{m}\right)$, and the real part is taken componentwise. 
A.2. Attractor-Bifurcation Theorem. For the attractor-bifurcation theory it suffices to assume that the nonlinear function $G_{\lambda}$ in Eq. (A.2) is a bounded $C^{r}$ map $(r \geq 1)$ from $H_{\alpha}$ into $H$ for some $\alpha \in[0,1)$, and that $G_{\lambda}$ satisfies the asymptotic estimate

$$
G_{\lambda}(w)=o\left(\|w\|_{H_{\alpha}}\right), \quad|w| \rightarrow 0
$$

uniformly in $\lambda$.

We use the following definitions.

Definition A.1. (i) $A$ set $\Sigma \subset H$ is a (positive) invariant set of Eq. (A.2) if $S_{\lambda}(t) \Sigma=\Sigma$ for any $t \geq 0$. (ii) An invariant set $\Sigma \subset H$ of $E q$. (A.2) is an attractor if $\Sigma$ is compact and there exists a neighborhood $U \subset H$ of $\Sigma$ such that $\lim _{t \rightarrow \infty} \operatorname{dist}_{H}\left(w_{\lambda}\left(t ; w_{0}\right), \Sigma\right)=0$ for any $w_{0} \in U$. (iii) The largest open set $U$ satisfying the above condition is the basin of attraction of $\Sigma$.

Definition A.2. (i) A solution $\left(w_{\lambda}, \lambda\right)$ of Eq. (A.2) bifurcates from $\left(0, \lambda_{0}\right)$ if there exists a sequence of invariant sets $\left\{\Sigma_{n}\right\}$ of $E q$. (A.2) with $0 \notin \Sigma_{n}$ such that $\lim _{n \rightarrow \infty} \max _{w \in \Sigma_{n}}|w|=0$ and $\lim _{n \rightarrow \infty} \operatorname{dist}\left(\lambda_{n}, \lambda_{0}\right)=$ 0. (ii) If the invariant sets $\Sigma_{n}$ are attractors of Eq. (A.2), then the bifurcation is called an attractor bifurcation. (iii) If the invariant sets $\Sigma_{n}$ are attractors and are homotopy equivalent to an m-dimensional sphere $S^{m}$, then the bifurcation is called an $S^{m}$-attractor bifurcation.

The solution $\left(w_{\lambda}, \lambda\right)$ of Eq. (A.2) bifurcates from $\left(0, \lambda_{0}\right)$ to an attractor $\mathcal{A}_{\lambda}$ as $\lambda$ passes through the critical value $\lambda_{0}$. The following theorem characterizes the attractor $\mathcal{A}_{\lambda}$ and the nature of the bifurcated solutions.

Theorem A.2 (Attractor-Bifurcation Theorem). If $m>1$, then $\mathcal{A}_{\lambda}$ has the following properties:

(1) $\mathcal{A}_{\lambda}$ is connected and $\operatorname{dim} \mathcal{A}_{\lambda} \in[m-1, m]$.

(2) $\mathcal{A}_{\lambda}$ is the limit of a sequence of m-dimensional annuli $\left\{M_{i}\right\}_{i}$ with $M_{i+1} \subset M_{i}$ for $i=1,2, \ldots$; in particular, if $\mathcal{A}_{\lambda}$ is a finite simplicial complex, then $\mathcal{A}_{\lambda}$ has the homotopy type of $S^{m-1}$.

(3) For any $w_{\lambda} \in \mathcal{A}_{\lambda}, w_{\lambda}$ can be expressed as

$$
w_{\lambda}=W_{\lambda}+z_{\lambda}, \quad W_{\lambda} \in E_{1}, \quad z_{\lambda}=o\left(\left\|W_{\lambda}\right\|_{H}\right) .
$$

(4) There is an open set $U \subset H$ with $0 \in U$ such that $\mathcal{A}_{\lambda}$ attracts $U \backslash \Gamma$, where $\Gamma$ is the stable manifold of $w=0$ with codimension $m$. 


\section{REFERENCES}

[1] Benson, D. L., P. K. Maini, and J. A. Sherratt, Unravelling the Turing Bifurcation Using Spatially Varying Diffusion Coefficients, J. Math. Biol. 37, 381-417, 1998.

[2] Bollerman, P., A. van Harten, and G. Schneider, On the Justification of the G-L Approximation. In: Nonlinear Dynamics and Pattern Formation in the Natural Environment, A. Doelman and A. van Harten (eds.), Longman, 1995, pp. 20-36.

[3] Cross, M. C., And P. C. Hohenberg, Pattern Formation outside of Equilibrium, Rev. Mod. Phys. 65, 851-1112, 1993.

[4] Dulos, E., J. Boissonade, J. J. Perraud, B. Rudovics, and P. De KepPER, Chemical Morphogenesis: Turing Patterns in an Experimental Chemical System, Acta Biotheoretica 44, 249-261, 1996.

[5] De Kepper, P., E. Dulos, J. Boissonade, A. De Wit, G. Dewel, and P. Borckmans, Reaction-Diffusion Patterns in Confined Chemical Systems, J. Stat. Phys. 101, 495-508, 2000.

[6] Eckhaus, W. The Ginzburg-Landau Manifold Is an Attractor, J. Nonlinear Science 3, 329-348, 1993.

[7] Henry, D., Geometric Theory of Semilinear Parabolic Equations, Lecture Notes in Mathematics, Vol. 840, Springer-Verlag, Berlin, 1981.

[8] Hoyle, R. Pattern Formation, An Introduction to Methods, Cambridge University Press, 2006.

[9] Iron, D., J. Wei, And M. Winter, Stability Analysis of Turing Patterns Generated by the Schnakenberg Model, J. Math. Biol. 49 (4), 358-390, 2004.

[10] Ma, T. AND S. WANG, Bifurcation Theory and Applications, World Scientific, Singapore, 2005.

[11] Ma, T. AND S. WANG, Bifurcation and Stability of Superconductivity, J. Math. Phys. 46, 095112:1-31, 2005.

[12] Maini, P. K., The Impact of Turing's Work on Pattern Formation in Biology, Mathematics Today 40(4), 140-141, 2004.

[13] Murray, J. D., Mathematical Biology II, 3rd ed., Springer-Verlag, 2003.

[14] Page, K. M., P. K. Maini, And N. A. M. Monk, Pattern Formation in Spatially Heterogeneous Turing Reaction-Diffusion Models, Physica D 181, 80101, 2002.

[15] Page, K. M., P. K. Maini, And N. A. M. Monk, Complex Pattern Formation in Reaction-Diffusion Systems with Spatially Varying Diffusion Coefficients, Physica D 202 95-115, 2005.

[16] Price, C. B., P. WambacQ, And A. Oosterlinck, Communications, Speech and Vision, IEEE Proceedings - Communications I 137(3), 136-145, 1990.

[17] Schnakenberg, J., Simple Chemical Reaction Systems with Limit Cycle Behavior, J. Theor. Biol. 81, 389-400, 1979.

[18] Schneider, G., Global Existence via Ginzburg-Landau Formalism and Pseudo-Orbits of G-L Approximations, Comm. Math. Phys. 164, 157-179, 1994.

[19] Schneider, G., Nonlinear Diffusive Stability of Spatially-Periodic SolutionsAbstract Theorem and Higher Space Dimensions, Tohoku Math. J. 8, 159-167, 1998. 
[20] Turing, A., The Chemical Basis of Morphogenesis, Philos. Trans. Roy. Soc. London B 237, 37-52, 1952.

[21] van Harten, A. On the Validity of the Ginzburg-Landau Equation, J. Nonlinear Science 1, 397-422, 1991.

[22] Wollkind, D. J., V. S. Manoranjan, and L. Zhang, Weakly Nonlinear Stability Analyses of Prototype Reaction-Diffusion Model Equations, SIAM Rev. 36, 176-214, 1994.

[23] Yochelis, A., And A. Garfinkel Front Motion and Localized States in an Asymmetric Bistable Activator-Inhibitor System with Saturation, Phys. Rev. E 77, 035204(R), 2008.

[24] Yochelis, A., Y. Tintut, L. L. Demer, and A. Garfinkel The Formation of Labyrinths, Spots and Stripe Patterns in a Biochemical Approach to Cardiovascular Calcification, New J. Phys. 10, 055002, 2008.

The submitted manuscript has been created in
part by the UChicago Argonne, LLC, Operator
of Argonne National Laboratory ("Argonne") un-
der Contract No. DE-AC02-06CH11357 with the
U.S. Department of Energy. The U.S. Government
retains for itself, and others acting on its behalf,
a paid-up, nonexclusive, irrevocable worldwide li-
cense in said article to reproduce, prepare deriv-
ative works, distribute copies to the public, and
perform publicly and display publicly, by or on
behalf of the Government.

(HGK) Mathematics and Computer Science Division, Argonne National Laboratory, Argonne, IL 60439; Current address: Division of Mathematical Sciences, National Science Foundation, Arlington, VA 22230

E-mail address: kaper@mcs.anl.gov; hkaper@nsf.gov

(SW) Department of Mathematics, Indiana University, Bloomington, IN 47405

E-mail address: showang@indiana.edu

(MY) Department of Mathematics, Indiana University, Bloomington, IN 47405

E-mail address: myari@indiana.edu 